\title{
Computing the Conjugacy of Invariant Tori for Volume-Preserving Maps*
}

\author{
Adam M. Fox ${ }^{\dagger}$ and James D. Meiss $\ddagger$
}

\begin{abstract}
In this paper we implement a numerical algorithm to compute codimension-one tori in threedimensional, volume-preserving maps. A torus is defined by its conjugacy to rigid rotation, which is in turn given by its Fourier series. The algorithm employs a quasi-Newton scheme to find the Fourier coefficients of a truncation of the series. We show that this method converges for tori of two example maps by continuation from an integrable case, and discuss the scaling of computational resources required for accurate computations. We demonstrate that the growth of the largest singular value of the derivative of the conjugacy predicts the threshold for the destruction of the torus. We use these singular values to examine the mechanics of the breakup of tori, identifying its onset with the formation of "spires" or "streaks" in the local singular values on the tori. These are analogous to the gaps in cantori of two-dimensional twist maps.
\end{abstract}

Key words. invariant tori, volume-preserving maps, KAM theory, transport barriers, sprial mean

AMS subject classifications. 37J15, 37J40, 70K43, 70K55, 70H12

DOI. $10.1137 / 15 \mathrm{M} 1022859$

1. Introduction. In this paper we will study families of maps $f_{\varepsilon}$ on $M=\mathbb{T}^{2} \times \mathbb{R}$ of the form

$$
\begin{aligned}
& x^{\prime}=x+\Omega(z)-\varepsilon g_{1}(x, z, \varepsilon) \bmod 1 \\
& z^{\prime}=z-\varepsilon g_{2}(x, z, \varepsilon)
\end{aligned}
$$

where $x \in \mathbb{T}^{2}$ are period-one angles and $z \in \mathbb{R}$ represents the action, and $\left(x^{\prime}, z^{\prime}\right) \in \mathbb{T}^{2} \times \mathbb{R}$ represents the image point. Maps of this form arise in the study of many natural phenomena including incompressible fluids [PF88, FO88a, CFP96], magnetic field lines [TH85], granular mixing [MLO07], and celestial mechanics [LS94].

Invariant tori play a prominent role in the dynamics of maps such as (1). Most notably, when $\varepsilon=0$ every orbit lies on a two-torus $\mathcal{T}_{z_{0}}=\mathbb{T}^{2} \times\left\{z_{0}\right\}$ on which the dynamics is simply a rigid rotation, i.e., for each $t \in \mathbb{Z}$,

$$
\left(x_{t}, z_{t}\right)=f_{0}^{t}\left(x_{0}, z_{0}\right)=\left(x_{0}+\omega t, z_{0}\right), \quad \omega=\Omega\left(z_{0}\right) ;
$$

such maps are integrable.

\footnotetext{
* Received by the editors May 26, 2015; accepted for publication (in revised form) by H. Osinga January 5, 2016; published electronically March 23, 2016. This research was supported in part by NSF grant DMS-1211350. This work utilized the Janus supercomputer, which is supported by the NSF grant CNS-0821794 and the University of Colorado Boulder.

http://www.siam.org/journals/siads/15-1/M102285.html

${ }^{\dagger}$ Department of Mathematics, Western New England University, Mailbox H-5122, Springfield, MA 01119 (Adam.Fox@wne.edu). The work of this author was also supported by Western New England University.

${ }^{\ddagger}$ Department of Applied Mathematics, University of Colorado, UCB-526, Boulder, CO 80309-0526 (James. Meiss@ colorado.edu).
} 
We will assume that (1) is an exact volume-preserving map, i.e., that there exists a one form $S$ such that $f_{\varepsilon}^{*} \alpha-\alpha=d S$ with respect to the two form $\alpha=z d x_{1} \wedge d x_{2}$. Exactness is a necessary condition for the existence of rotational invariant tori when $\varepsilon \neq 0$. We say a torus is rotational if it is homotopic to $\mathcal{T}_{z_{0}}$. The rotational tori of (1) are fundamentally important as they form barriers to transport: orbits that begin on one side cannot cross to the other [FP88, App. B].

KAM theory [CS90, Xia92] guarantees the persistence of a Cantor set of rotational tori in exact volume-preserving, near-integrable maps of the form (1), assuming that $g_{1}, g_{2}$, and $\Omega$ are smooth enough, and the frequency map $\Omega: \mathbb{R} \rightarrow \mathbb{R}^{2}$ satisfies a Kolmogorov nondegeneracy assumption

$$
\operatorname{rank}\left(\Omega, D \Omega, D^{2} \Omega, \ldots\right) \geq 2
$$

The persistent tori have rotation vectors $\omega$ that are Diophantine,

$$
\omega \in \mathcal{D}_{s}=\left\{\omega \in \mathbb{R}^{2} \mid \exists c>0 \text { s.t. }|p \cdot \omega-q|>c|p|^{-s}, \quad \forall(p, q) \in \mathbb{Z}^{3} \backslash\{0\}\right\}
$$

for some $s \geq 2$. It is important to note that, unlike the Hamiltonian case, this theory does not predict the persistence of a torus with a given rotation vector for a given map $f_{\varepsilon}$, but just a Cantor set of Diophantine tori when $\varepsilon$ is small enough.

Although KAM theory does guarantee the persistence of some tori for small $\varepsilon$, it does not say anything about what happens when $\varepsilon=\mathcal{O}(1)$. John Greene [Gre79] developed the first quantitative method to study the persistence of tori for the case of two-dimensional, areapreserving maps. He conjectured that periodic orbits in the neighborhood of an invariant circle should be stable; indeed, a sequence of periodic orbits should limit upon a circle only if they remain stable in the limit. Conversely, if the limit of a family of periodic orbits is unstable, then the invariant circle should no longer exist. This method is known as Greene's residue criterion [Gre79]. In [FM13], we extended this method to the volume-preserving case; however, our generalization is only applicable to maps with reversing symmetries and symmetric tori.

In this paper, we will compute the tori directly, rather than a sequence of approximating periodic orbits. An advantage of this method is that the map need not be reversible. In section 2 we describe a Fourier-based scheme, analogous to that developed for symplectic maps by de la Llave et al. [HdlL07, HdlLS12, FdlLS09], to compute the embedding for an invariant torus with given rotation vector in volume-preserving maps of the form (1). Although this algorithm can be used to study invariant tori in any volume-preserving system, it is easiest to apply to maps, such as (1), that have an integrable limit. Indeed, since the method is iterative, it requires a good initial guess for a torus. The integrable tori at $\varepsilon=0$ provide a simple starting point for continuation.

Numerically, each torus is represented by an embedding $k: \mathbb{T}^{2} \rightarrow M$ that conjugates the dynamics to rigid rotation with a given Diophantine rotation vector $\omega$; the particular rotation vectors that we study are described in section 3 . We will find tori for two examples: the standard, reversible map studied in [FM13], and the well-known ABC map [FO88b], a nonreversible system that commonly arises in fluid mechanics; see section 4 . 
In either case, as $\varepsilon$ grows, we will see that the conjugacy $k$ appears to lose smoothness as the torus nears destruction. To visualize this, we study the local singular values of the $3 \times 2$ matrix $D k$ in section 5 . A point on a torus where the singular values are large is strongly deformed under the embedding. We will see that the spikes in the singular values are organized into spires or streaks that appear to correspond to the incipient formation of holes in the torus, mirroring the behavior known to occur when invariant circles in area-preserving twist maps are destroyed and replaced by cantori [Mei15].

We will show that divergence of the largest singular value of the matrix $D k$ can be used to estimate the threshold for destruction of a torus, by analogy with norm-based methods used for area-preserving maps [CdlL10a, CdlL10b, FM14]. In section 5.2 we exploit this divergence to estimate the critical parameter, $\varepsilon_{c r}$, for destruction of a torus. For the standard, reversible map, the close agreement between the two sets of results provides significant numerical evidence of the validity of both the extension of Greene's criterion developed in [FM13] and the singular value method presented here. Computations of critical parameters are also given for the ABC map. Since this map is not reversible, periodic orbits cannot be easily computed, and thus applying Greene's criterion is not practical. Our method does not require reversibility so we are able to compute tori and their breakdown for a variety of parameters.

2. The quasi-Newton algorithm. In this section we obtain a quasi-Newton scheme to find rotational invariant tori for a family of maps of the form (1). Although this algorithm can be applied to arbitrary volume-preserving maps with codimension-one tori, we restrict our focus to three-dimensional families of the form (1).

The method is based on a Fourier series expansion for the conjugacy to rigid rotation. To guarantee convergence of these series we assume that $f_{\varepsilon}$ is analytic. Moreover, as explained more below, we suppose that the map depends upon a set of auxiliary parameters $\lambda$, denoting the new map by $f_{\lambda, \varepsilon}$, and assume that $f$ is a $C^{2}$ function of $\lambda$.

The goal is to compute an embedding $k: \mathbb{T}^{2} \rightarrow M$ to a rotational torus (should one exist), i.e.,

$$
\mathcal{T}=\left\{\left(k_{x}(\theta), k_{z}(\theta)\right) \mid \theta \in \mathbb{T}^{2}\right\},
$$

where the angle components, $k_{x}$, are two dimensional, the action component, $k_{z}$, is a scalar. For a rotational torus, the angle components have degree one and the action component is periodic:

$$
k(\theta+m)=k(\theta)+(m, 0)^{T} \quad \forall m \in \mathbb{Z}^{2} .
$$

The dynamics on the torus are assumed to have a given rotation vector $\omega$, that is, its dynamics are conjugate to the rigid translation $T_{\omega}(\theta) \equiv \theta+\omega$,

$$
f_{\lambda, \varepsilon} \circ k=k \circ T_{\omega}
$$

This is equivalent to the commutative diagram illustrated in Figure 1.

Note that solutions of (4), if they exist, are not unique: given a solution $k(\theta)$, then $k(\theta+\chi)$ is also a solution $\forall \chi \in \mathbb{R}^{2}$. However, when $\omega$ is incommensurate, this is the only nonuniqueness for continuous conjugacies. 


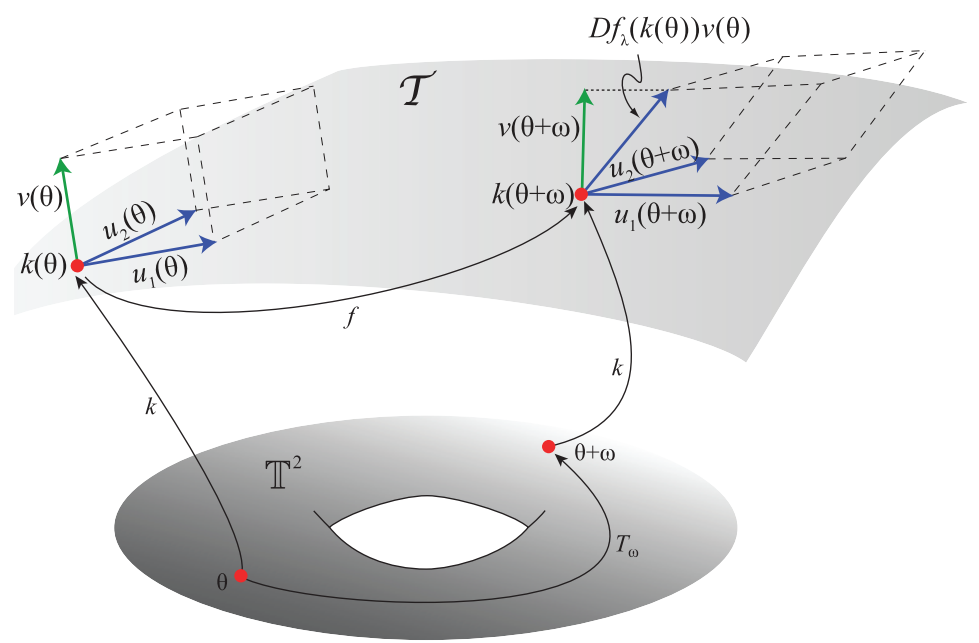

Figure 1. A visualization of the commutative diagram for automatic reducibility, and the iteration of the tangent, $\left(u_{1}, u_{2}\right)$, and normal, $v$, vectors by the linearization $D f$. The dashed parallelepipeds have unit volume.

Lemma 1 (see [FM14]). If $k \in C^{0}\left(\mathbb{T}^{2}, M\right)$ solves (4) for an incommensurate rotation vector $\omega$, then every other continuous solution of (4) for the same invariant torus is of the form $k(\theta+\chi)$ for some $\chi \in \mathbb{T}^{2}$.

The auxiliary parameters $\lambda$ in $f_{\lambda, \varepsilon}$ are needed in order to fix the rotation number of the torus. The point is that when $\varepsilon=0$, the image of the frequency map $\Omega$ in (1) is a curve $\{\Omega(z) \mid z \in \mathbb{R}\} \subset \mathbb{R}^{2}$; thus in this case, only orbits with rotation vectors on this curve will exist. To guarantee the existence of a torus with a chosen $\omega$ it is necessary to add a parameter to the frequency map; we suppose $\Omega$ depends upon $\delta \in \mathbb{R}$ in such a way that

$$
(z ; \delta) \mapsto \Omega(z ; \delta)
$$

is a diffeomorphism onto $\mathbb{R}^{2}$. One can view $\delta$ as a parameter that selects a particular curve and $z$ as a parameter that selects a point along this curve in order that $\Omega(z ; \delta)=\omega$.

To extend this to $\varepsilon \neq 0$, let $\lambda=(\delta, p)$ denote the set of parameters, where the average action,

$$
p=\int_{\mathbb{T}^{2}} k_{z}(\theta) d \theta \equiv\left\langle k_{z}\right\rangle,
$$

represents the position along the image of the true frequency map. More precisely, for $y \equiv z-p$, (1) becomes

$$
f_{\lambda, \varepsilon}:\left\{\begin{array}{l}
x^{\prime}=x+\Omega(y+p ; \delta)-\varepsilon g_{1}(x, y+p, \varepsilon) \bmod 1 \\
y^{\prime}=y-\varepsilon g_{2}(x, y+p, \varepsilon)
\end{array}\right.
$$

However, we continue to use $z$ as the action variable in our presentation rather than $y$. In this case, the parameter $p$ does not appear in the map, but, instead appears in the conjugacy:

$$
k(\theta)=\left(\begin{array}{l}
\theta \\
0
\end{array}\right)+\left(\begin{array}{l}
0 \\
p
\end{array}\right)+\tilde{k}(\theta) .
$$


By (6) and a choice of the shift in angle, we can assume that the periodic part of the conjugacy has zero average: $\langle\tilde{k}\rangle=0$. This function is represented by a Fourier series

$$
\tilde{k}(\theta)=\sum_{j \in \mathbb{Z}^{2} \backslash\{0\}} \hat{k}_{j} e^{2 \pi i j \cdot \theta}
$$

with coefficients $\hat{k}_{j}=\hat{k}_{-j}^{*} \in \mathbb{C}^{3}$.

The idea is that for every $\omega \in \mathcal{D}_{2}$ and for small enough $\varepsilon$, there should exist parameters $\lambda=\lambda(\varepsilon)$ and a conjugacy (7) such that the map (1) has a torus $\mathcal{T}=k\left(\mathbb{T}^{2}\right)$ on which the dynamics is conjugate to rigid rotation with rotation vector $\omega$. This is the analogue of the parameterization method developed for the symplectic case [HdlL07, HdlLS12, FdILS09]. ${ }^{1}$ Our goal in this paper is to compute these tori.

2.1. Automatic reducibility. To implement the iterative algorithm to find $k$, we begin with a guess $(k(\theta), \lambda)$ that is an approximate solution of $(4)$ :

$$
f_{\lambda, \varepsilon} \circ k-k \circ T_{\omega}=e .
$$

The iteration then proceeds by assuming that there is a nearby conjugacy $k+\Delta$ that satisfies (4) for a nearby map $f_{\lambda+\zeta, \varepsilon}$. Expanding (4) then gives (we drop the subscripts on $f_{\lambda, \varepsilon}$ for the remainder of this exposition to avoid the notational clutter)

$$
f(k(\theta))+D f(k(\theta)) \Delta(\theta)+D_{\lambda} f(k(\theta)) \zeta-k(\theta+\omega)-\Delta(\theta+\omega)=\mathcal{O}\left(\Delta^{2}, \zeta^{2}\right),
$$

where $D f$ and $D_{\lambda} f$ indicate the derivatives of $f$ with respect to the state variables and parameters $\lambda$, respectively. Neglecting second-order terms, using (9), and reordering then gives an iterative equation,

$$
\Delta(\theta+\omega)-D f(k(\theta)) \Delta(\theta)=e(\theta)+D_{\lambda} f(k(\theta)) \zeta,
$$

that, as we see below, can be viewed as determining $(\Delta, \zeta)$. If a solution is found, then $k+\Delta$ is an approximate conjugacy for the map $f_{\lambda+\zeta, \varepsilon}$ in the sense of satisfying (9) with a new, presumably smaller, error $e$. This new map and error can then be used to obtain a new correction by solving (10) again.

We will see in section 4 that, in practice, the $L^{2}$-norm of the error, $\|e\|_{2}$ goes to zero with successive iterations, but not as fast as a classical Newton method. The reason for this is that there are certain conditions that must be satisfied in order to solve (10). These solvability conditions can be imposed by a proper choice of the parameter increment $\zeta$, but it is consistent with the iterative nature of our algorithm to implement them approximately.

The operator acting on $\Delta$ on the left-hand side of (10) is called a cohomology operator in [HdlLS12], and in order to solve (10), its right-hand side must be in the range of this operator. Inversion of the cohomology operator on its range will then determine $\Delta$ up to elements of the kernel. However a direct inversion would be numerically expensive. It is better to partially

\footnotetext{
${ }^{1}$ While we developed the numerical algorithm independently, we learned during the preparation of this paper that that Blass and de la Llave are also extending these ideas to the volume-preserving case [BdlL15]. Their research is focused on providing a rigorous justification of a method similar to ours.
} 
diagonalize the operator through a process called automatic reducibility in [HdlLS12]. The idea is that there exists a change of variables $\Delta(\theta)=M(\theta) w(\theta)$, so that in terms of the new vector $w(\theta)$, (10) takes the form

$$
w(\theta+\omega)-S(\theta) w(\theta)=h(\theta)+G(\theta) \zeta .
$$

As we will see, $M(\theta)$ can be chosen to be a unimodular matrix, $S(\theta)$ a special upper-triangular matrix, and

$$
\begin{aligned}
G(\theta) & =M^{-1}(\theta+\omega) D_{\lambda} f(k(\theta)), \\
h(\theta) & =M^{-1}(\theta+\omega) e(\theta) .
\end{aligned}
$$

Since we do not invert the cohomology operator, this algorithm is not truly a Newton method, hence we refer to it as quasi-Newton.

So that (11) is equivalent to (10) the matrices $M$ and $S$ must solve the matrix system

$$
D f(k(\theta)) M(\theta)=M(\theta+\omega) S(\theta) .
$$

This can be done by choosing the columns of $M$ to be a set of tangent and normal vector fields of the (approximate) torus $\mathcal{T}$. Note that if $k$ were an exact conjugacy, then differentiation of (4) would give

$$
D f(k(\theta)) D_{\theta} k(\theta)=D_{\theta} k(\theta+\omega),
$$

which is the statement that the columns of $D_{\theta} k=\left(u_{1} u_{2}\right)$, vector fields tangent to $\mathcal{T}$, are invariant under $f$. For the implementation of the algorithm, $k$ will never be an exact conjugacy, thus it will only approximately satisfy (14). Nevertheless, we may use (14) in the Newton iteration (10) incurring error only at second order. We assume that the guess for the conjugacy, $k(\theta)$, is selected so these two tangent vector fields are uniformly independent:

$$
\left\|u_{1}(\theta) \times u_{2}(\theta)\right\| \geq c>0
$$

where " $\times$ " is the standard cross product and $\|\cdot\|$ the Euclidean norm. Then the columns of $M$ are selected to be these two tangent vector fields and a scaled normal:

$$
\begin{aligned}
M(\theta) & \equiv\left(\begin{array}{ll}
u_{1}(\theta) \quad u_{2}(\theta) \quad v(\theta)
\end{array}\right), \\
u_{1}(\theta) & \equiv D_{\theta_{1}} k(\theta), \\
u_{2}(\theta) & \equiv D_{\theta_{2}} k(\theta) \\
v(\theta) & \equiv \frac{u_{1}(\theta) \times u_{2}(\theta)}{\left\|u_{1}(\theta) \times u_{2}(\theta)\right\|^{2}} .
\end{aligned}
$$

Under the nondegeneracy assumption, $M$ is well-defined, $\operatorname{det} M(\theta) \equiv 1$, and

$$
M^{-1}(\theta)=\left(u_{2}(\theta) \times v(\theta) \quad v(\theta) \times u_{1}(\theta) \quad u_{1}(\theta) \times u_{2}(\theta)\right)^{T} .
$$

Note that (14) then implies that (neglecting the error)

$$
D f(k(\theta)) M(\theta)=\left(u_{1}(\theta+\omega) \quad u_{2}(\theta+\omega) \quad D f(k(\theta) v(\theta)) .\right.
$$


Combining this with (13) shows that $S=\left(\hat{e}_{1} \hat{e}_{2} s\right)$, i.e., the first two columns of $S$ are trivially the unit basis vectors. Moreover, because $M$ is unimodular and $f$ is volume preserving, the determinant of (13) implies that $S_{33}=s_{3}=1$ as well. Thus $S$ is a special upper-triangular matrix:

$$
S(\theta)=\left(\begin{array}{ccc}
1 & 0 & s_{1}(\theta) \\
0 & 1 & s_{2}(\theta) \\
0 & 0 & 1
\end{array}\right)=M^{-1}(\theta+\omega) D f(k(\theta)) M(\theta) .
$$

Performing the matrix multiplication on the right determines the last two components of $S$ :

$$
\begin{aligned}
& s_{1}(\theta)=u_{2}(\theta+\omega) \times v(\theta+\omega) \cdot D f(k(\theta)) v(\theta), \\
& s_{2}(\theta)=v(\theta+\omega) \times u_{1}(\theta+\omega) \cdot D f(k(\theta)) v(\theta) .
\end{aligned}
$$

The three rows of (11) now yield skew coupled equations for the components of the vector $w$,

$$
\begin{aligned}
& w_{1}(\theta+\omega)-w_{1}(\theta)=h_{1}(\theta)+G_{1}(\theta) \cdot \zeta+s_{1}(\theta) w_{3}(\theta), \\
& w_{2}(\theta+\omega)-w_{2}(\theta)=h_{2}(\theta)+G_{2}(\theta) \cdot \zeta+s_{2}(\theta) w_{3}(\theta), \\
& w_{3}(\theta+\omega)-w_{3}(\theta)=h_{3}(\theta)+G_{3}(\theta) \cdot \zeta
\end{aligned}
$$

where $s$ is defined by (18), and $h$ and $G_{i}$ - the $i$ th row of $G$-by (12).

These three equations can be solved easily in Fourier space. Indeed, each is of the form of a cohomology equation

$$
w \circ T_{\omega}-w=h,
$$

that is diagonalized by Fourier transformation. It is not hard to see that if $h$ is analytic and $\omega$ is Diophantine (3), then $w$ is analytic [Mos66] and its Fourier coefficients are

$$
\hat{w}_{j}=\frac{\hat{h}_{j}}{e^{2 \pi i j \cdot \omega}-1}, j \neq 0,
$$

provided that $h$ satisfies the solvability condition

$$
\hat{h}_{0}=\int_{\mathbb{T}^{2}} h(\theta) d \theta=0,
$$

i.e., that its average vanishes. Since the average, $\hat{w}_{0}=\langle w\rangle$, is in the kernel of the cohomology operator, it can be chosen freely.

2.2. Solvability. Beginning with a guess for the conjugacy $k$ and parameters $\lambda$, we compute the error $e$ from (9) and the vector fields $u_{1}, u_{2}$, and $v$ from (16). Now $G$ and the modified error $h$ can be computed from (12), and $s$ from (18). At this point the cohomology equations (19)-(21) can be solved using (22), under the assumption that the solvability conditions

$$
\begin{aligned}
\left\langle h_{1}\right\rangle+\left\langle G_{1}\right\rangle \cdot \zeta+\left\langle s_{1} w_{3}\right\rangle & =0, \\
\left\langle h_{2}\right\rangle+\left\langle G_{2}\right\rangle \cdot \zeta+\left\langle s_{2} w_{3}\right\rangle & =0, \\
\left\langle h_{3}\right\rangle+\left\langle G_{3}\right\rangle \cdot \zeta & =0,
\end{aligned}
$$


are satisfied. These equations illustrate the importance of the parameters $\lambda$-they allow us to control averages and ensure the solvability of the cohomology equations.

To implement these conditions, we adopt the simplest, approximate method: we simply ignore the third condition above! Even though this assumption is not generally true for an approximate conjugacy, we observe numerically that it does not prevent the convergence of the method; indeed when $h \rightarrow 0$, so does the error induced by this inconsistency; see Figure 3(b) in section 4 . The average of $w_{3}$ may then be freely chosen. For simplicity, we set it to zero. At this point, the first and second conditions of (23) can be used as conditions to fix $\zeta$, under the assumption that $\left\langle G_{1}\right\rangle$ and $\left\langle G_{2}\right\rangle$ are independent. This condition is satisfied for (1), at least at $\varepsilon=0$, because then $k(\theta)=(\theta, p), M=I$,

$$
\left(\begin{array}{l}
G_{1}(\theta) \\
G_{2}(\theta)
\end{array}\right)=D_{\lambda} \Omega(p ; \delta)
$$

and $\Omega$ was assumed to be a diffeomorphism in $\lambda=(\delta, p)$. As we will see in section 4 ignoring the third solvability condition is not completely benign: it makes the iteration converge slower than quadratically. It may also be possible to ignore the first or second solvability condition rather than third - we did not explore this possibility.

The averages of $w_{1}$ and $w_{2}$ are arbitrary; these averages add contributions $u_{1}(\theta)\left\langle w_{1}\right\rangle$ and $u_{2}(\theta)\left\langle w_{2}\right\rangle$ to $k$ contributing to a shift along the torus that corresponds to the nonuniqueness implied by Lemma 1 . We set $\left\langle w_{1}\right\rangle=\left\langle w_{2}\right\rangle=0$ for simplicity. Even so, $\left\langle u_{i}(\theta) w_{i}(\theta)\right\rangle \neq 0$, so horizontal shifts still occur.

To conclude our description of the method: once $w$ is found, $k$ and $\lambda$ are updated by $k \rightarrow k+M(\theta) w(\theta)=k+\Delta(\theta)$ and $\lambda \rightarrow \lambda+\zeta$. Note that even though we set $\langle w\rangle=0$, the average of the update, $\left\langle\Delta_{3}\right\rangle$, may be nonzero, adding a vertical shift to $k$. Thus to ensure that $p$ represents the average action, we replace $p \rightarrow p+\left\langle\Delta_{3}\right\rangle$ and $k_{z} \rightarrow k_{z}-\left\langle\Delta_{3}\right\rangle$. This is a cosmetic change that simply keeps the interpretation of $p$ fixed.

2.3. Continuation and antialiasing. To obtain good initial guesses for $k$, we use continuation from the integrable limit $\varepsilon=0$, where $k(\theta)=(\theta, p)$ is trivial. Each of cohomology equations (19)-(21) is solved by using the fast Fourier transform, for $N \times N$ modes, i.e.,

$$
w(\theta)=\sum_{j_{1}, j_{2} \in\left(-\frac{N}{2}, \frac{N}{2}\right]} \hat{w}_{j_{1}, j_{2}} e^{2 \pi i j \cdot \theta} .
$$

We typically begin with $N=2^{7}$ for the first step from $\varepsilon=0$. Since the number of modes is finite, and the maps are nonlinear, aliasing errors occur when the spectrum wraps around due to periodicity in mode number. This error can be ameliorated by application of an antialiasing filter [Tre00]. We simply set the Fourier modes $\hat{w}_{j}=0$ whenever $\|j\|_{\infty}>J=\frac{1}{4} N$ for each solution of the cohomology equations (22).

The iteration is declared successful if the $L^{2}$-norm of the error (9) reaches the tolerance, $\|e\|_{2}<10^{-12}$ (using double precision arithmetic); otherwise it is declared to fail if $\|e\|_{2}>10^{3}$ or after ten Newton iterations. Since the functions are represented by finite Fourier series, the rate of decrease in the error sometimes slows before reaching the desired tolerance. If the error fails to decrease by at least $5 \%$ after two successive iterations, the algorithm exits, rejecting 
the solution. The nondegeneracy condition (15) is checked when the tangent vectors (16) are computed: the algorithm would fail if $\left\|u_{1}(\theta) \times u_{2}(\theta)\right\|<10^{-6}$, however, this never occurred.

Continuation in $\varepsilon$ is performed beginning with an increment $\Delta \varepsilon$. The values of $(k, \lambda)$ at the integrable $\varepsilon=0$ limit are used as an initial guess for $\varepsilon=\Delta \varepsilon$, and linear extrapolation is then used to predict $(k, \lambda)$ for $\varepsilon=2 \Delta \varepsilon$. For each successive step, quadratic extrapolation is employed to estimate $(k, \lambda)$ for the new torus. This fixed step size and number of modes is used until the algorithm fails to converge within the specified tolerance. Upon this first failure, the step size $\Delta \varepsilon$ is halved and $N$ is doubled. The antialiasing filter is applied directly to the embedding $k(\theta)$ whenever this doubling occurs. At each subsequent failure to converge, the number of Fourier modes is again doubled and the step size is halved. To limit the computation time, the doubling of Fourier modes ceases upon reaching an upper limit, which we typically took to be $N_{\max }=2^{11}$. The algorithm also exits when convergence fails at a minimum specified $\Delta \varepsilon=\Delta \varepsilon_{\min }$.

The choice of initial increment $\Delta \varepsilon$ and threshold $\Delta \varepsilon_{\text {min }}$ depends on both the map being studied and the performance desired. Smaller choices of these parameters will yield more accurate results but will increase the runtime. Furthermore, if the tori being studied tend to be destroyed quickly, then smaller values of these parameters should be used to ensure accuracy. Conversely, if the tori are relatively robust, larger values may be used to reduce runtime without affecting accuracy. See section 4 for details.

3. Diophantine rotation vectors. Since the robust tori of standard KAM theory have Diophantine rotation vectors, it is useful to have a systematic method to select such vectors. As is well known, each basis of an algebraic field projects to a Diophantine vector; in particular, when $(\omega, 1) \in \mathbb{R}^{3}$ is a basis for a cubic algebraic field then $\omega \in \mathcal{D}_{2}$ [Cas57]. As in [FM13], we concentrate our initial studies on the cubic field $\mathbb{Q}(\sigma)$ generated by the real root

$$
\sigma \approx 1.3247179572447460
$$

of the polynomial $x^{3}-x-1$. This number, called the "spiral mean" by [KO86] and the "plastic" number by [Ste96], is the smallest cubic PV number: a root of a monic polynomial with exactly one root outside the unit circle. The vector $\left(\sigma^{2}, \sigma, 1\right)$ is an integral basis for $\mathbb{Q}(\sigma)$; consequently any integral basis can be obtained from this vector by application of a matrix in $G l(3, \mathbb{Z})$. Our initial investigation focuses on one such basis with

$$
\omega=\left(\sigma-1, \sigma^{2}-1\right)
$$

in the unit square. We also used this extensively in [FM13].

For each incommensurate $\omega \in \mathbb{R}^{2}$, the binary generalized Farey tree of [KO86] gives a sequence of rational approximations

$$
\omega_{\ell}=\frac{m_{\ell}}{n_{\ell}}, \quad\left(m_{\ell}, n_{\ell}\right) \in \mathbb{Z}^{2} \times \mathbb{N},
$$

where $\omega_{\ell} \rightarrow \omega$ as $\ell \rightarrow \infty$. The rotation vector (24) corresponds to the infinite binary path $l l \bar{r}$ on this tree, where $l$ and $r$ denote the symbols for "left" and "right" turns on the tree, respectively. For the vector $(24)$, this sequence is $\left(m_{\ell}, n_{\ell}\right)=\left(n_{\ell-1}, n_{\ell+2}, n_{\ell+3}\right)$, where the 
periods satisfy the three-step recursion relation

$$
n_{\ell+3}=n_{\ell+1}+n_{\ell}, \quad n_{0}=0, n_{1}=n_{2}=1 .
$$

These periods grow at the rate $n_{\ell} \sim \sigma^{\ell}$, and the approximations (25) converge to $\omega$ as $\omega_{\ell}-\omega \sim \sigma^{-3 \ell / 2}$.

The rotation vector (24) corresponds to the Farey path $l l r r r . \ldots=l l \bar{r}$, consisting of two left turns followed by infinitely many rights. In section 5 we will investigate a number of invariant tori with rotation vectors in $\mathbb{Q}(\sigma)$; they all will have paths with an infinite tail of $r$ 's. For more details, see [FM13].

4. Examples. As a first application, we compute tori for the "standard volume-preserving" map

$$
\begin{aligned}
& x^{\prime}=x+\Omega\left(z^{\prime} ; \delta\right), \\
& z^{\prime}=z-\varepsilon g(x),
\end{aligned}
$$

with the quadratic frequency map

$$
\Omega(z ; \delta)=\left(z+\gamma, \beta z^{2}-\delta\right),
$$

which is a special case of (1). This map, derived in [DM12], models the local behavior near any rank-one resonance for three-dimensional volume-preserving dynamics. We use $\delta$ as a distinguished parameter in (27); in particular note that for any $\beta$ and $\gamma$, the frequency map $\Omega: \mathbb{R}^{2} \rightarrow \mathbb{R}^{2}$ is a diffeomorphism so the nondegeneracy condition is satisfied. For each $\delta$, the image of $\Omega$ is a parabola with a vertical intercept controlled by $\delta$. As noted in section 2, we also use the average action (6) as a parameter. Thus for $(26), \lambda=(\delta, p)$. Following previous studies [FM13, Mei12], we let

$$
g(x)=a \sin \left(2 \pi x_{1}\right)+b \sin \left(2 \pi x_{2}\right)+c \sin \left(2 \pi\left(x_{1}-x_{2}\right)\right)
$$

be the force and choose a standard set of parameters

$$
a=b=c=1, \beta=2, \gamma=\frac{1}{2}(\sqrt{5}-1) .
$$

With these choices, (26) is an analytic diffeomorphism. Since $g$ is odd, it is also reversible, a fact that we exploited in [FM13] to compute periodic orbits and implement a generalization of Greene's residue criterion. There we determined the parameters at which an invariant torus is destroyed by computing the stability of a sequence of periodic orbits whose rotation numbers satisfy (25). Here we compare these computations with those of the conjugacy $k(\theta)$.

Note that when $\varepsilon=0$ the rotational torus $k(\theta)=(\theta, p)$ exists when $\Omega(p ; \delta)=\omega$, or equivalently, using the rotation vector (24), for the choice of parameters

$$
p=\sigma-1-\gamma, \quad \delta=\beta p^{2}+1-\sigma^{2} .
$$

Using this as the initial guess, we first increment $\varepsilon$ by $\Delta \varepsilon=0.001$ and apply the iterative method as discussed in section 2.3. The resulting computations for $2^{7} \times 2^{7}$ Fourier modes converge to within $\|e\|_{2}=1.1 \times 10^{-15}$ upon four Newton steps, requiring only three seconds on a laptop with a $2.4 \mathrm{GHz}$ Intel Core i5 and $4 \mathrm{~Gb}$ of memory. The convergence is equally rapid as $\varepsilon$ is incremented; for example, after ten increments, when $\varepsilon=0.01$, the algorithm converged within $\|e\|_{2}=5.6 \times 10^{-13}$ upon three iterations; the resulting embedded torus is 


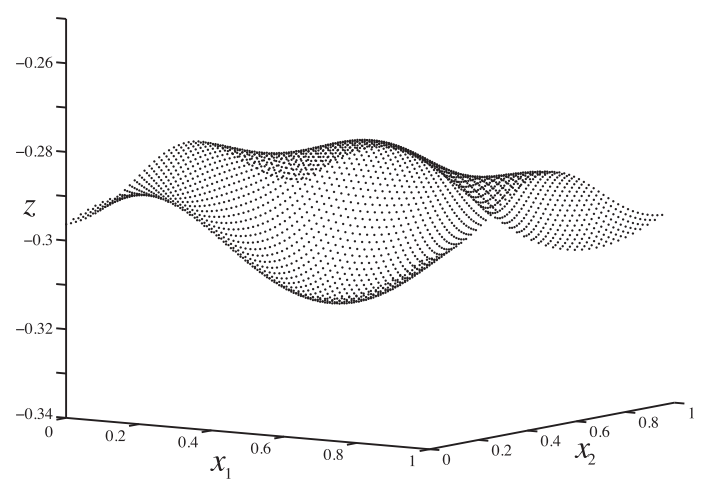

(a)

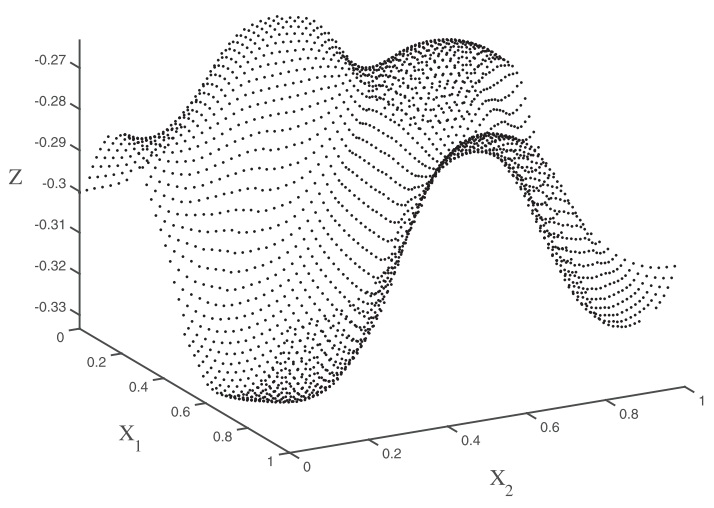

(b)

Figure 2. The embedded invariant torus for the standard volume-preserving map (26) with rotation vector $\left(\sigma-1, \sigma^{2}-1\right)$ at (a) $\varepsilon=0.01$ and $(\mathrm{b}) \varepsilon=0.02545$. Here $(\delta, p)=(-0.58269,-0.29331)$, and $(-0.58201,-0.29330)$, respectively. The points correspond to a uniform grid of 50 points for each angle.

shown in Figure 2(a). Fewer iterations are needed in this case because extrapolation provides a more accurate initial guess for the embedding. The algorithm first fails to converge within the specified tolerance when $\varepsilon=0.018$, triggering a decrease in $\Delta \varepsilon$ to $5 \times 10^{-4}$, and an increase in the number of Fourier modes to $2^{8} \times 2^{8}$. A second failure occurs at $\varepsilon=0.02250$, leading to a further increase in the number of Fourier modes and reduction in $\Delta \varepsilon$. The algorithm exited when $\Delta \varepsilon<\Delta \varepsilon_{\min }=10^{-6}$.

Although our algorithm is based on Newton's method, we observe that its convergence is not quadratic. Nevertheless, the convergence appears to be superlinear, as shown in Figure 3(a): the error after $j$ iterations decreases as

$$
\left\|e_{j}\right\|_{2} \sim c^{m^{j}}
$$

with $c<1$ and an exponent $m \approx 1$.4. The exponent $m$ decreases slightly with $\varepsilon$ independent of the number of modes used, implying that aliasing has little effect on the convergence rate. This subquadratic convergence is not unexpected. Ignoring the solvability condition in (21) causes an $\mathcal{O}(e)$ error in the iteration. Indeed, the Newton method converges to the torus at nearly the same rate that the neglected solvability condition for $w_{3}$ converges to zero, shown in Figure 3(b). As a test, we also implemented the full six-dimensional system needed to solve (19)-(21) and impose the condition $\langle M(\theta) w(\theta)\rangle=0$. Although more algorithmically cumbersome, the convergence was significantly more rapid, as shown in Figure 3(a), with exponent $m \approx 1.6$ in (30). However, application of the $6 \times 6$ system did not improve the final accuracy or the ability to compute tori for larger $\varepsilon$. Hence, below, we will use the numerically simpler, $2 \times 2$ approximate solvability condition.

The performance of the algorithm for the rotation vector (24) and different maximum values of Fourier modes, $N_{\max }$, was tested on a single node of the Janus supercomputer. Each node employs $24 \mathrm{~GB}$ of memory and contains two hex-core $2.8 \mathrm{GHz}$ Intel Westmere processors. The results are summarized in Table 1. As expected, when $N$ doubles the total memory required increases by a factor of four. The total runtime for larger $N_{\max }$ does not 


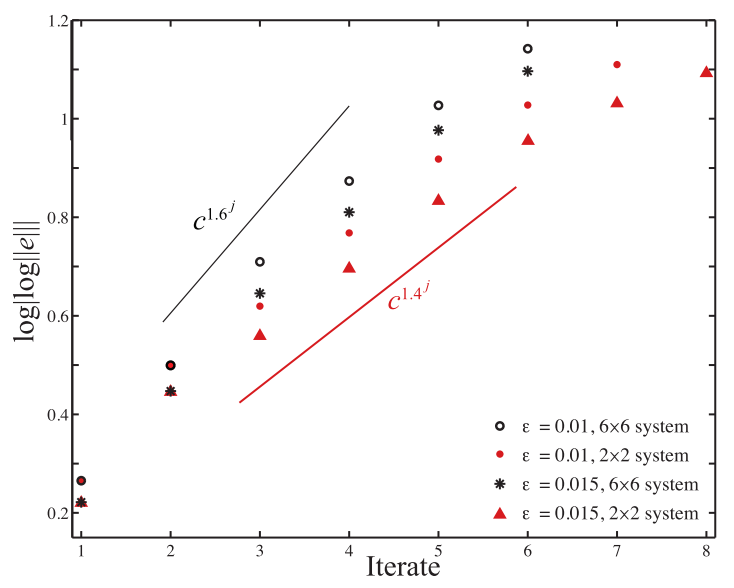

(a)

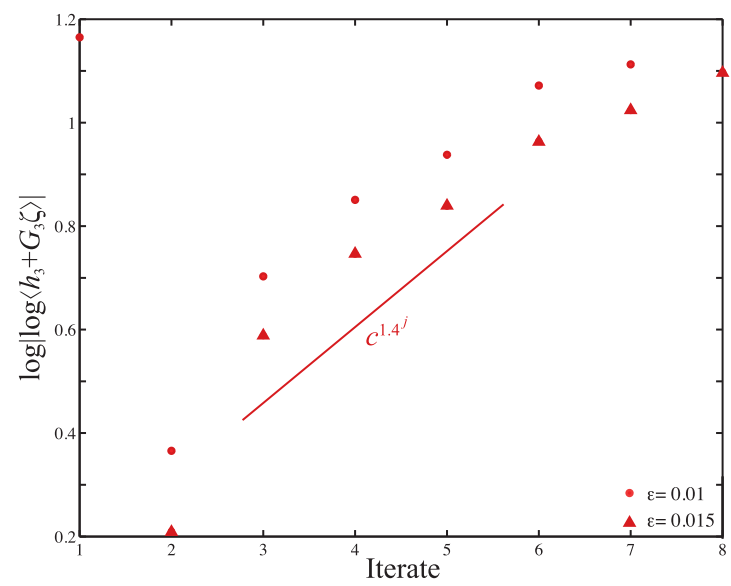

(b)

Figure 3. Errors in the computation of the spiral mean torus for (26) as a function of the Newton iterate, using the integrable torus as the initial guess, on a $\log |\log ()|$ scale. Note that the actual errors range from $10^{-10^{0.2}} \approx 0.026$ to $10^{-10^{1.2}} \approx 1.4(10)^{-16}$. (a) The $L^{2}$ error in the conjugacy (9) for the values of $\varepsilon$ and technique indicated. (b) Magnitude of the neglected solvability coefficient, $\left\langle h_{3}+G_{3} \zeta\right\rangle$, for the $2 \times 2$ solvability method; recall (21). Since this error goes to zero, the solvability condition is asymptotically satisfied.

Table 1

Performance data for the computation of the torus with spiral mean rotation number (24) in the standard volume preserving map (26). The estimates of $\varepsilon_{c r}$ are derived using the singular value method; see section 5.2 . The numbers in parentheses indicate the estimated error in the least significant digit of $\varepsilon_{c r}$. Greene's residue criterion (see [FM13]) estimates $\varepsilon_{\text {cr }}$ to be $0.02580(1)$.

\begin{tabular}{c|l|l|r|r|c}
\hline$N_{\max }$ & $\varepsilon_{\max }$ & $\varepsilon_{c r}$ & Time $(\min )$ & Memory $(\mathrm{MB})$ & $\left\|k_{x}\right\|_{2}$ \\
\hline $2^{9}$ & 0.0242 & $0.0253507(6)$ & 2.55 & 239 & $1.017 \times 10^{6}$ \\
$2^{10}$ & 0.0250 & $0.0259(1)$ & 10.05 & 1,043 & $9.132 \times 10^{6}$ \\
$2^{11}$ & 0.02545 & $0.02581(2)$ & 45.47 & 4,165 & $9.005 \times 10^{7}$ \\
$2^{12}$ & 0.02547 & $0.02581(3)$ & 104.73 & 16,653 & $3.780 \times 10^{8}$ \\
\hline
\end{tabular}

increase as fast since most of the iterations are still done with smaller values of $N$. Ultimately there was little difference in performance between $N_{\max }=2^{11}$ and $N_{\max }=2^{12}$ (see section 5.2 for further details), so we will use $N_{\max }=2^{11}$ throughout this paper unless otherwise noted.

The algorithm converges to the specified accuracy $\|e\|_{2}<10^{-12}$ for each $\varepsilon$ up to $\varepsilon_{\max }$; this last torus is shown in Figure 2(b) when $N_{\max }=2^{11}$. These $\varepsilon$ values are smaller than the critical value $\varepsilon_{c r}=0.0258$ that we previously computed using Greene's criterion [FM13]. The Sobolev seminorm of the embedding,

$$
\|k\|_{2}=\sum_{j \in\left(-\frac{N}{2}, \frac{N}{2}\right]^{2}}\left(2 \pi\|j\|_{2}\right)^{2}\left\|\hat{k}_{j}\right\|_{2}^{2}
$$

appears to approach infinity as the torus nears destruction. The growth of this norm, shown for the $x$ component of $k$ in Table 1, provides further evidence that the tori we computed are near critical. For more general rotation numbers and maps, we observe that the algorithm typically converges for $\varepsilon$ values within $5 \%$ of the previously computed $\varepsilon_{c r}$ when $N_{\max }=2^{11}$. However, 


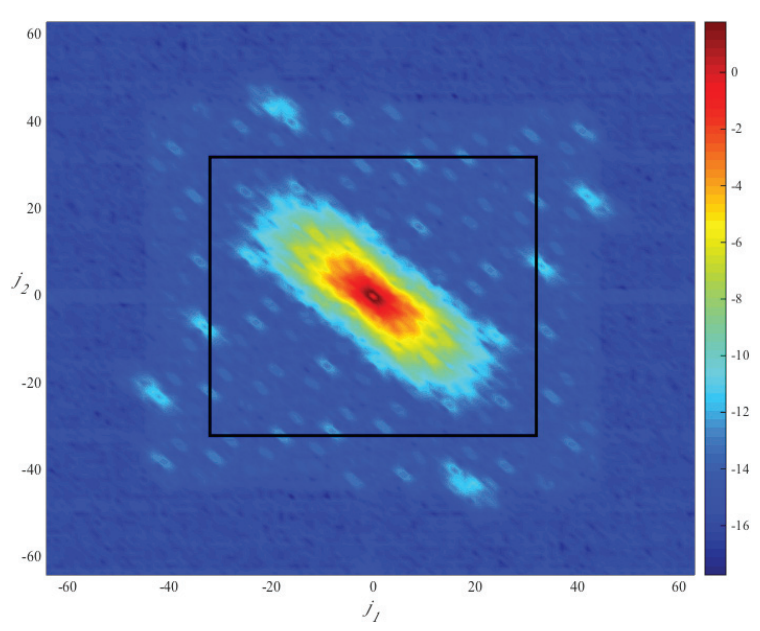

(a)

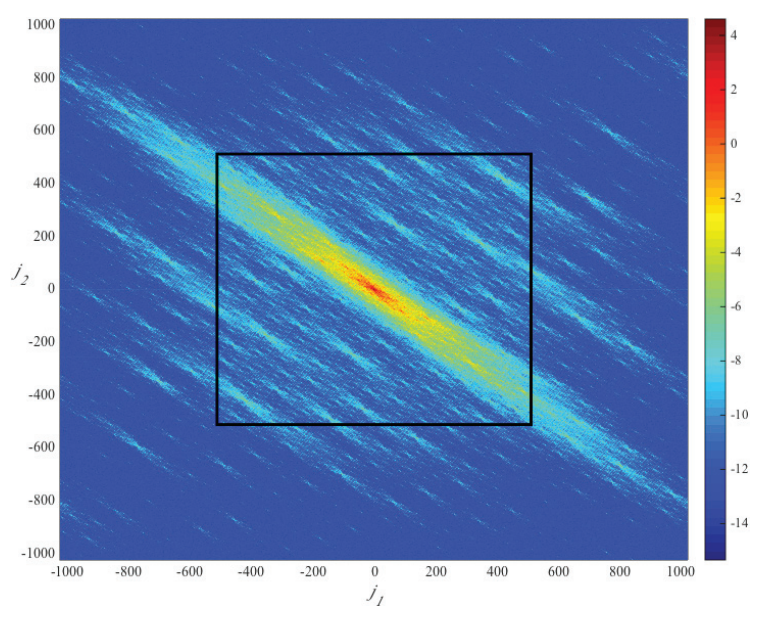

(b)

Figure 4. Fourier spectrum of $k_{x}$ for the spiral mean torus at (a) $\varepsilon=0.01$ for $N=2^{7}$ and (b) $\varepsilon=0.02545$ for $N=2^{11}$. The $x$ and $y$ axes indicate the first and second mode number, respectively, of the Fourier series. In each case the antialiasing filter is indicated by the black box: all modes outside the box are zeroed upon inversion of the cohomology operator for $w$.

in a few cases (where the singular values of the conjugacy grow rapidly; see section 5.2) the algorithm stopped when $\varepsilon$ was $10-12 \%$ below $\varepsilon_{c r}$. The main difficulty in getting closer to $\varepsilon_{c r}$ is that the width of the spectrum of the conjugacy grows with $\varepsilon$; see Figure 4. Initially, when the torus is far from criticality, such as in panel (a), no Fourier modes with significant amplitude fall within the region subject to antialiasing - the area outside the black square. However, for $\varepsilon=0.02545$, shown in panel (b) of the figure, significant modes do lie in this region and even extend to the maximum mode number. This leads to larger error.

As a validation of the new method, we compared these computations with those in [FM13] that used periodic orbits to approximate the torus. We computed the sequence $\left.\mathcal{O}_{n_{\ell}}=\left\{\left(x_{t}, z_{t}\right) \mid 0 \leq t<n_{\ell}\right)\right\}$ of period $n_{\ell}$ orbits for $n_{\ell}=1, \ldots, 7739$, with rotation numbers (25) and with the symmetry $x_{0}=0$. To compare $\mathcal{O}_{n}$ to the computed embedding, we first used a root finder to determine an angle $\theta_{t}$ for which the horizontal distance $\left|x_{t}-k_{x}\left(\theta_{t}\right)\right|=0$ for each $t \in[0, n)$. The average vertical distance

$$
d(n)=\frac{1}{n} \sum_{t=0}^{n-1}\left|z_{t}-k_{z}\left(\theta_{t}\right)\right|
$$

then provides a measure of the closeness of these orbits; see Figure 5. The bounding line in the figure shows that $d(n)$ decreases geometrically with an upper bound $n^{-1.5}$. Since this exponent is the same as that for the convergence of $\omega_{\ell}$ to $\omega$ (recall section 3 ), this provides strong numerical evidence that the limiting torus computed by the two methods is identical. A second measure of the closeness of the orbits comes from the parameter $\delta$. The values, $\delta_{\ell}$, for the periodic orbits converge to the $\delta$ value computed using the Fourier method at the same rate and agree to the same accuracy as the average vertical distance. Finally, (27) implies that the average action $p$ of the torus is $\omega_{1}-\gamma=\sigma-1-\gamma$, which the Fourier method accurately computes to 16 digits. 


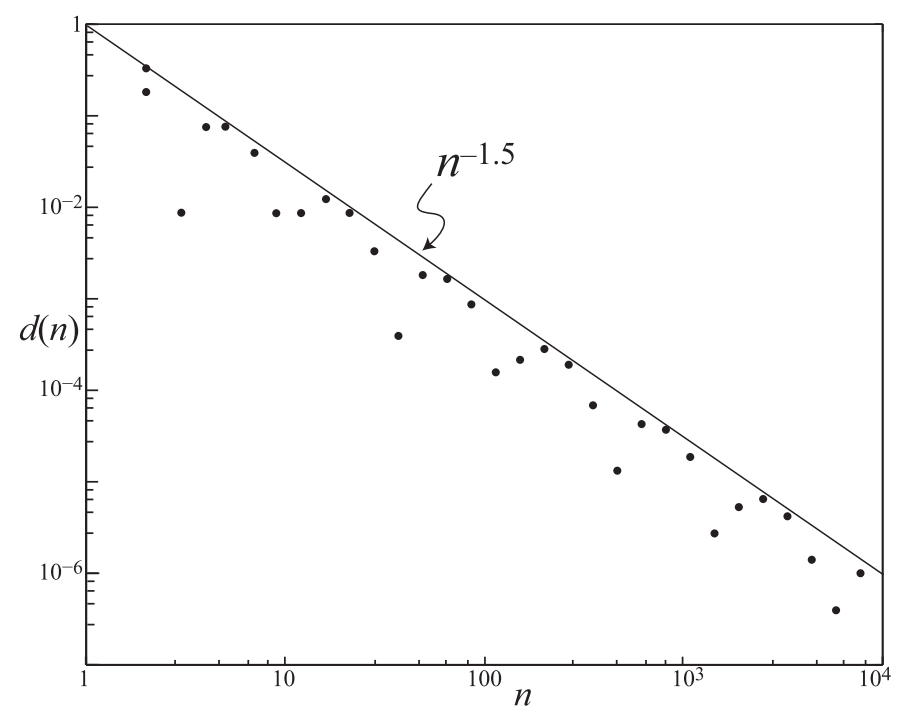

Figure 5. The mean vertical distance (31) between the period- $n_{\ell}$ approximating periodic orbits up to $n_{32}=$ 7739 (recall section 3), and the invariant torus with rotation vector (24) for $\varepsilon=0.01$ (recall Figure 2). The line shown is $d=n^{-1.5}$.

Since the map (26) is reversible, we expect the conjugacy to satisfy certain symmetry conditions; see [FM14, App. D]. In particular, the conjugacy for the angles $k_{x}(\theta)$ must be odd about the point $\varphi=-\left\langle k_{x}-\theta\right\rangle$ while the conjugacy of the action $k_{z}(\theta)$ must be even about $\varphi+\frac{1}{2} \omega$. These symmetries lead to requirements on the Fourier modes of the conjugacies (see $[$ FM14, (42)]) that provide an additional measure of the accuracy of the Fourier method). For the $\left(\sigma-1, \sigma^{2}-1\right)$ torus at $\varepsilon=0.01$ we find $\varphi=(1.11,-1.44) \times 10^{-6}$ and the identities hold up to an $L^{\infty}$ error of less than $3 \times 10^{-12}$ for each component, comparable to the overall accuracy of the computation of $k$.

As a second test of the Fourier method, we now consider the oft-studied ABC map,

$$
\begin{aligned}
& x^{\prime}=x+\frac{A}{2 \pi} \sin (2 \pi z)+\frac{C}{2 \pi} \cos (2 \pi y), \\
& y^{\prime}=y+\frac{B}{2 \pi} \sin \left(2 \pi x^{\prime}\right)+\frac{A}{2 \pi} \cos (2 \pi z), \\
& z^{\prime}=z+\frac{C}{2 \pi} \sin \left(2 \pi y^{\prime}\right)+\frac{B}{2 \pi} \cos \left(2 \pi x^{\prime}\right) .
\end{aligned}
$$

This map is an analytic, volume-preserving diffeomorphism on $\mathbb{T}^{3}$ for each $A, B, C \in \mathbb{R}[\mathrm{FO} 88 \mathrm{a}$, FO88b, FP88, MW99]. It can be thought of as a leapfrog (note the $x^{\prime}$ and $y^{\prime}$ on the right-hand side of (32)) Euler integrator for the Arnold-Beltrami-Childress flow [DFG $\left.{ }^{+} 86\right]$.

The map (32) is a near-integrable map of the form (1) if, for example, $C=\varepsilon$ and $B=B(\varepsilon)$ with $B(0)=0$. Since the invariant tori of (32) have constant $z=p$ when $\varepsilon=0$, we think of $z$ as an actionlike variable, and of $x$ and $y$ as angles. For this case the frequency map is

$$
\Omega(z ; A)=\frac{A}{2 \pi}(\sin (2 \pi z), \cos (2 \pi z)) .
$$

We treat $A$ as an essential parameter, similar to $\delta$ in (26), so that $\Omega: \mathbb{S}^{1} \times(0, \infty) \rightarrow \mathbb{R}^{2} \backslash\{0\}$ is a diffeomorphism. As before we use $p=\left\langle k_{z}\right\rangle$ as the second parameter, setting $\lambda=(A, p)$. 


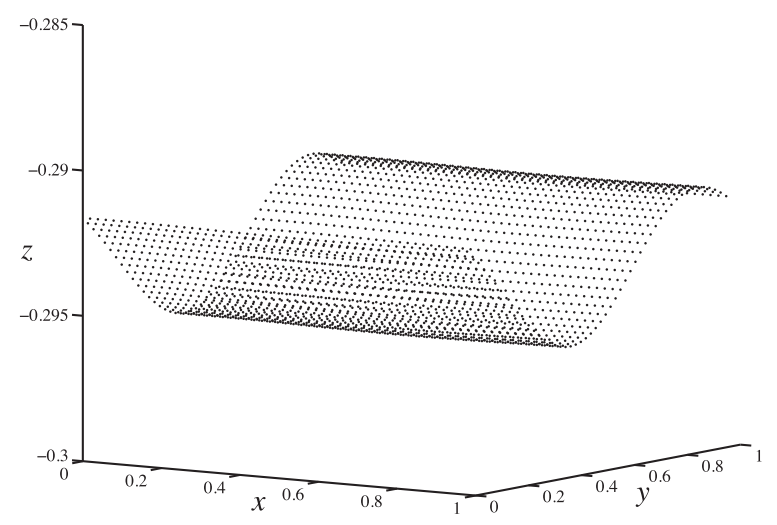

(a)

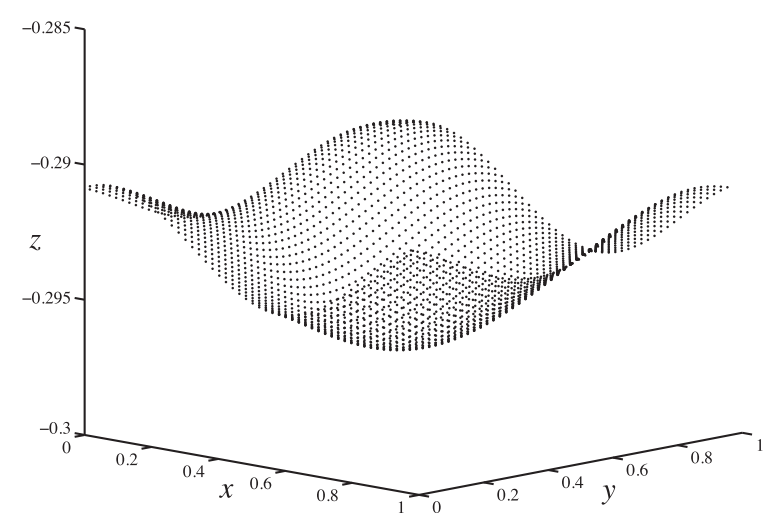

(b)

Figure 6. Invariant tori with rotation vector (24) for the $A B C$ map (32): (a) $B=\varepsilon^{2}$ at $\varepsilon=0.02$, and $A=5.1640$; (b) with $B=2 \varepsilon$ at $\varepsilon=0.01$ and $A=5.1638$. The points represent a uniform grid of 50 points for each $\theta$.

Here we consider two cases for $B(\varepsilon): B=\varepsilon^{2}$ and $B=2 \varepsilon$. When $B=\varepsilon^{2} \ll \varepsilon$ the dynamics of $y$ and $z$ essentially decouple from that of $x$, and the variation of the torus in the $x$-direction is very small. The spiral mean torus, with $\omega$ given by (24), is shown for this case at $\varepsilon=0.02$ in Figure 6(a). However, when $B \sim \varepsilon$, such as in Figure 6(b), where $B=2 \varepsilon$ with $\varepsilon=0.01$, the torus deforms along all dimensions. In both cases, the quasi-Newton scheme converges superlinearly, as it did for the standard volume-preserving map. When $B=\varepsilon^{2}$ we set $\Delta \varepsilon=0.005$ and when $B=2 \varepsilon, \Delta \varepsilon=0.0025$. In both cases, $\Delta \varepsilon_{\min }=0.001 \Delta \varepsilon$.

5. Detecting critical tori. The breakup of tori in volume-preserving maps is far less understood than the breakup of invariant circles in two-dimensional twist maps. In particular, neither Aubry-Mather nor anti-integrability theory have been generalized to nonsymplectic, volume-preserving systems to prove the existence of remnant tori. ${ }^{2}$ Since a codimension-one invariant torus is a barrier to transport, one implication of the destruction of a torus is the existence of crossing orbits [Mei12]. In our previous study, we observed that the period- $n_{\ell}$ orbits of (26) for large level $\ell$, lose stability at what appears to be the same parameter, $\varepsilon=\varepsilon_{c r}$, from which these crossing orbits are born [FM13]. For $\varepsilon>\varepsilon_{c r}$ the periodic orbits persist, however, they are increasingly unstable and difficult to compute. The density approximated by these periodic orbits becomes highly nonuniform and they appear to approximate a remnant torus analogous to the cantorus of area-preserving twist maps. We do not know, however, the topology of these remnants - if they indeed exist. It seems plausible that there are remnants that correspond to tori with one or more deleted orbits of open disks: topologically they would be Sierpinski curves.

In this section we show that the formation of holes is also suggested by our computations of the conjugacy of subcritical tori. We visualize the local stretching on the torus by computing the singular values of the $3 \times 2$ matrix $D k(\theta)$. The squared, largest singular value of $D k(\theta)$,

\footnotetext{
${ }^{2}$ Anti-integrable theory has been used to show the existence of nontrivial invariant sets for some third-order difference equations [JLM08].
} 


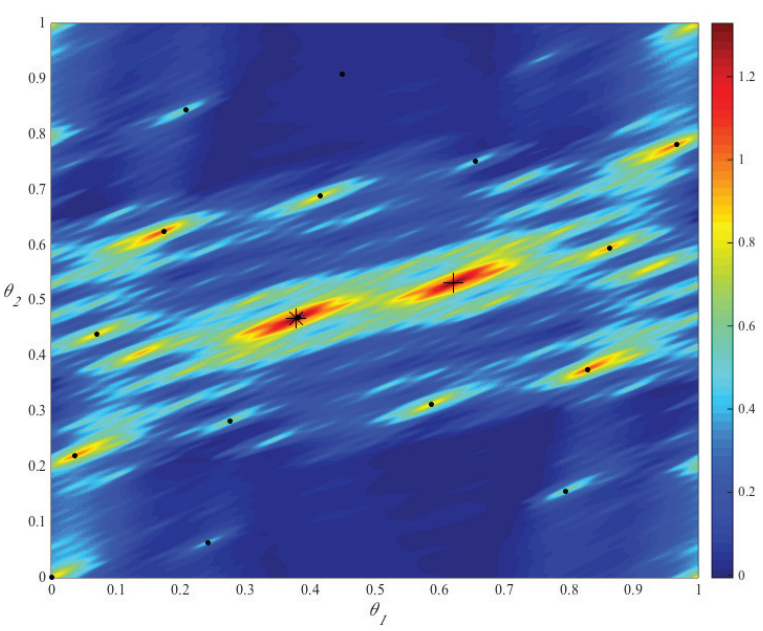

(a)

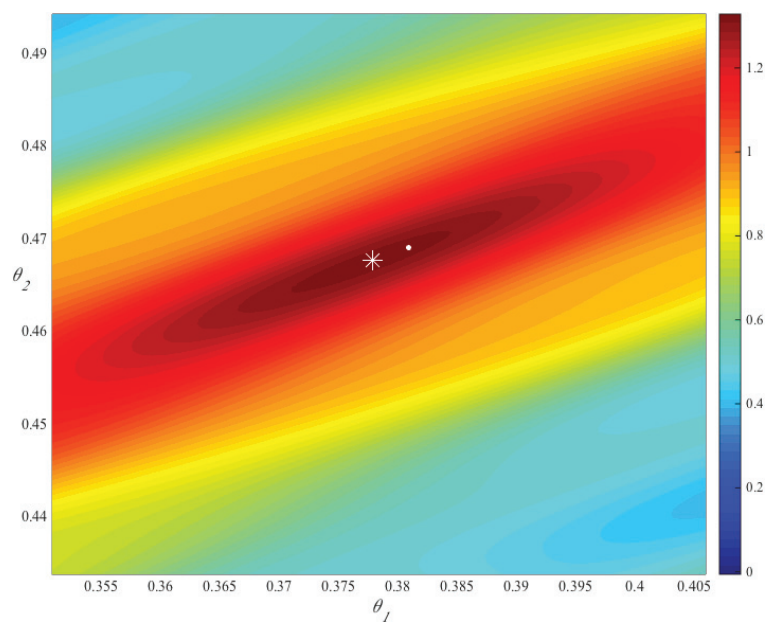

(b)

Figure 7. Singular values for the near-critical torus with $\omega=\frac{1}{5}\left(-3 \sigma^{2}+4 \sigma+1,-\sigma^{2}+3 \sigma+2\right)$ (Farey path llrrl $\bar{r})$, of the standard volume-preserving map (26). The color scale is $\log \mathcal{S}(\theta)$, the maximum value of $\mathcal{S}$ is indicated by the black plus, the images and preimages of this maximum are indicated by the black dots, and the peak of the symmetric spire is indicated with the black star. (a) The torus at $\varepsilon=0.0168125$. The maxima of $\mathcal{S}$ occur at $\theta_{m}=(0.6221,0.5322)$ and $(1,1)-\theta_{m}$. (b) A magnification of the second spire. The white star indicates the peak while the white dot is an iterate of the peak of the largest spire

i.e., the largest eigenvalue of

$$
\left(D k^{T} D k\right)_{i j}=u_{i} \cdot u_{j}
$$

(recall (16)), is denoted $\mathcal{S}(\theta)$. When the torus is smooth, $\mathcal{S}(\theta)$ is finite, but as the torus begins to tear apart, the singular values at some $\theta$ values appear to grow indefinitely.

Rapid growth of the largest singular value signals the destruction of the torus. We demonstrate in this section how the blowup of singular values, a proxy for the loss of smoothness of the conjugacy, can be used to estimate $\varepsilon_{c r}$. This method is similar to the Sobolev norm methods of [CdlL10a, CdlL10b, FM14].

5.1. Near-critical conjugacies. The breakup of tori in the standard volume-preserving map appears to follow a pattern similar to that for circles in area-preserving twist maps [FM14]. For the latter, breakup corresponds to the formation of one or more gaps at some point on the circle. Incipient gaps correspond to spikes in the derivative $D k$. The images of these largest gaps form a bi-infinite family upon iteration forward and backward in time; each such family is called a "hole."

For two-tori, however, there are interesting variations in the geometry and location of regions where $\mathcal{S}(\theta)$ is large. One example for the standard volume preserving map (26) is shown in Figure 7. For this case the spikes in $\mathcal{S}(\theta)$ are localized to patches, which we refer to as spires. These always seem to occur in symmetric pairs about $\theta=\left(\frac{1}{2}, \frac{1}{2}\right)$, due to the reversing symmetry of the map. In every observed torus, the spires correspond to a single hole, i.e., the orbit of the position of the maximum of $\mathcal{S}$ lies at the center of all of the spires. For example the two symmetric maxima in Figure $7(\mathrm{a})$ are six iterates apart and the remaining, smaller 


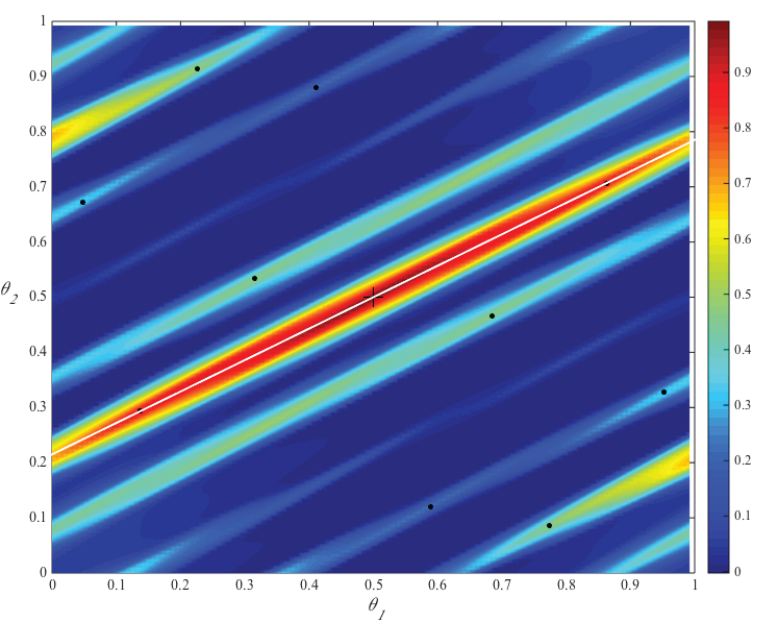

(a)

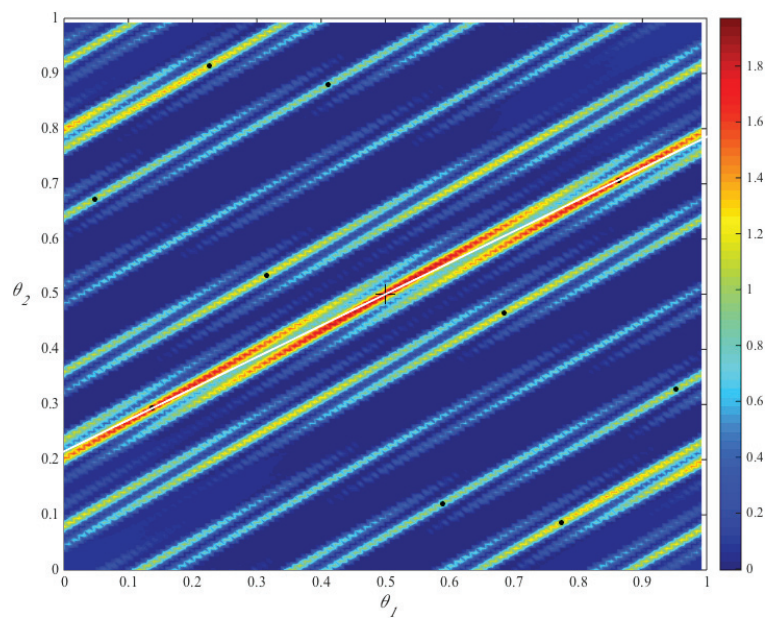

(b)

Figure 8. Singular value $\mathcal{S}(\theta)$ for the torus with $\omega=\left(2 \sigma^{2}-\sigma-2, \sigma^{2}-\sigma\right)$ (Farey path llrl $\bar{r}$ ), in the standard volume-preserving map (26). The color scale is $\log \mathcal{S}(\theta)$, the maximum value of $\mathcal{S}$ is indicated by the black plus, and images and preimages of the maximum are indicated by the black dots. (a) For $\varepsilon=0.009$ there is a dominant streak aligned with the rotation vector $\omega$ (white line). (b) For $\varepsilon=0.0099375$, the main streak has broken up and is no longer aligned with $\omega$. Several other smaller streaks have formed, each is centered on an image of the location of the maximum singular value.

peaks lie on the same orbit. Although the orbit of the maximum of $\mathcal{S}$ does not land exactly on the peak of the symmetric spire (see Figure 7(b)), this is almost certainly due to sampling issues. Indeed, as $N_{\max }$ was increased the distance between these points declined. In some cases the largest spires are separated by many iterates. For example, when the rotation vector has the Farey path $l l r r r l \bar{r}$, the symmetric spires are separated by 47 iterations.

The peaks in the singular values more commonly develop into elongated patches that we call streaks. These may be due to the merging of symmetric spires such as those seen in Figure 7. The torus in Figure 8(a) exhibits a dominant streak about the maximal singular value that is aligned with the rotation vector $\omega$; we have, however, observed other alignments. As this torus approaches criticality its main streak breaks apart, forming smaller streaks whose centers lie along the orbit of the primary peak; see Figure 8(b).

To understand the breakup of tori in the ABC map (32) we must first recognize that the dynamics are significantly altered whenever $B \equiv 0$. In this case the $y$ and $z$ dimensions are completely decoupled from the angle $x$, hence the dynamics of the map are essentially two dimensional with a quasi-periodically forced third dimension; recall Figure 6(a). The two-dimensional map in angle $y$ and action $z$ is area preserving, and its inverse,

$$
f_{y z}^{-1}(y, z)=\left(y-\frac{A}{2 \pi} \cos \left(2 \pi z^{\prime}\right), z-\frac{C}{2 \pi} \sin (2 \pi y)\right),
$$

can be analyzed with anti-integrability theory for fixed $A$, e.g., [Aub95]. In this case (35) has the potential $V_{y z}(y)=\frac{C}{4 \pi^{2}} \cos (2 \pi y)$, and the theory predicts that the largest gap forms around the maximum at $y=0$, and this is what is observed; see Figure 9(a). Similarly, when $C=0$ (not shown) the main streak forms along the maximum of the potential $V_{x z}(x)=\frac{B}{4 \pi^{2}} \sin (2 \pi x)$ 


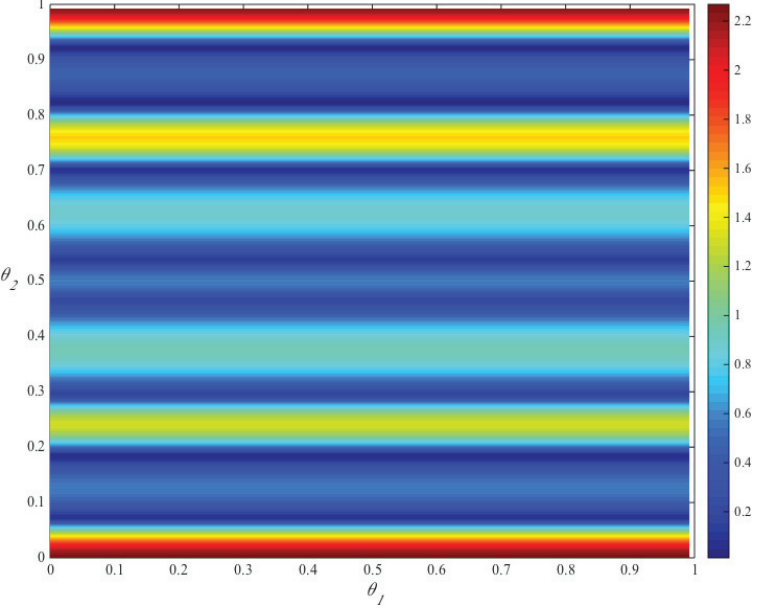

(a)

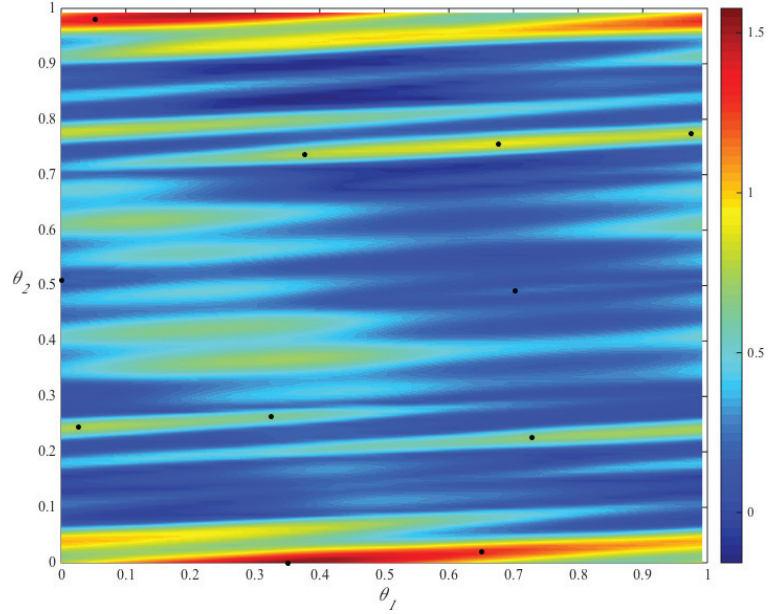

(b)

Figure 9. (a) Logarithm of $\mathcal{S}(\theta)$ for the spiral mean (Farey path llर्r) torus of the $A B C$ map with $C=$ 0.2040625 and $B=0$. The main streak forms near the line $\theta_{2}=0$. (b) The same torus with $C=\varepsilon=0.1475$ and $B=\varepsilon^{2}$. Iterates of the location of the maximum are indicated by black dots.

for the area-preserving map for $(x, z)$, that is, along $x=\frac{1}{4}$. These patterns continue to hold when $B$ is either asymptotically smaller or larger than $C$. For example, when $B=\varepsilon^{2} \ll C$, a streak forms along the line $x=0$, similarly to the $B=0$ torus; see Figure 9(b). As $\varepsilon$ grows, the perturbation in the $y$ direction increases, and this streak deforms. Additional streaks also form along the orbit of the main streak.

When $B=\mathcal{O}(C)$ there is no consistent pattern to the breakup of the tori. In some cases we see streaks form near $x=\frac{1}{4}$ or $y=0$, while in others spires appear to arise at the intersection of the maxima and minima of the potentials $V_{y z}(y)$ and $V_{x z}(x)$; see Figure 10.

5.2. The singular value method. A family of volume-preserving maps (1) $f_{\lambda, \varepsilon}$ has a curve $f_{\lambda(\varepsilon), \varepsilon}$ along which an invariant torus with Diophantine rotation vector $\omega$ exists for all $\varepsilon \in\left[0, \varepsilon_{c r}(\omega)\right]$. To estimate $\varepsilon_{c r}$ we use the blowup of the squared singular value $\mathcal{S}(\theta)$. Indeed, we observe that as $\varepsilon \rightarrow \varepsilon_{c r}$

$$
\|\mathcal{S}(\theta)\|_{\infty} \sim \frac{\kappa}{\left(\varepsilon_{c r}-\varepsilon\right)^{\beta}}
$$

see Figure 11. This fit works for both of the maps that we studied as well as for tori with a variety of rotation vectors. However, the exponent $\beta$ varies with the map, as can be seen in the figure, as well as the rotation vector, as we will show below.

We used the last ten steps of the continuation in $\varepsilon$ to estimate the parameters $\varepsilon_{c r}, \beta$, and $\kappa$ in (36) using the built-in nonlinear least squares algorithm of MATLAB. We obtained a rough estimate of the error in $\varepsilon_{c r}$ from the penultimate ten continuation steps; however, this variation in the estimate of $\varepsilon_{c r}$ appears to often underestimate the true error. We call this technique the "singular value method" for computing $\varepsilon_{c r}$.

The results of the singular value method for the spiral mean torus with $\omega=\left(\sigma-1, \sigma^{2}-1\right)$ were shown in Table 1 . The estimate of $\varepsilon_{c r}$ converges as $N_{\max }$ is increased-indeed, there is 


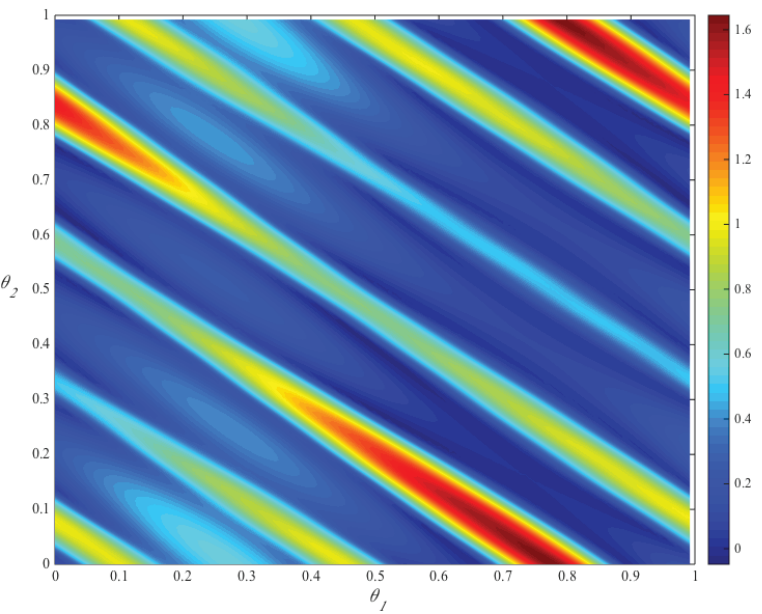

(a)

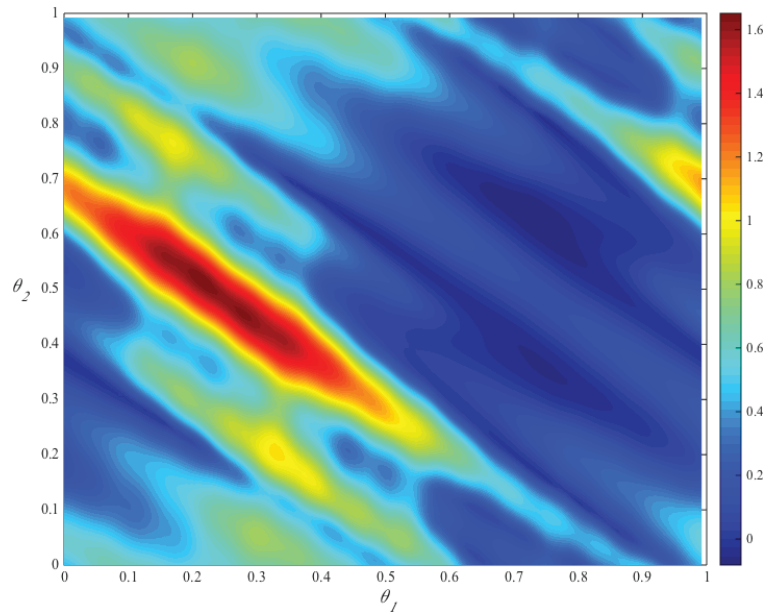

(b)

Figure 10. Logarithm of $\mathcal{S}(\theta)$ for tori of the $A B C$ map with $C=\varepsilon$ and $B=2 \varepsilon$. (a) The near-critical torus with $\omega=\frac{1}{7}\left(5 \sigma^{2}-3 \sigma+1,6 \sigma^{2}-5 \sigma-3\right)$ (Farey path rrllrl $\bar{r}$ ) at $\varepsilon=0.018125$. The main spire has formed near $\theta=(0.75,0)$, the intersection of the maximum of $V_{y z}$ and the minimum of $V_{x z}$. (b) The near-critical llrrl $\bar{r}$ torus at $\varepsilon=0.0325$. The main spire has formed near $\theta=(0.25,0.5)$, the intersection of the minimum of $V_{y z}$ and the maximum of $V_{x z}$.

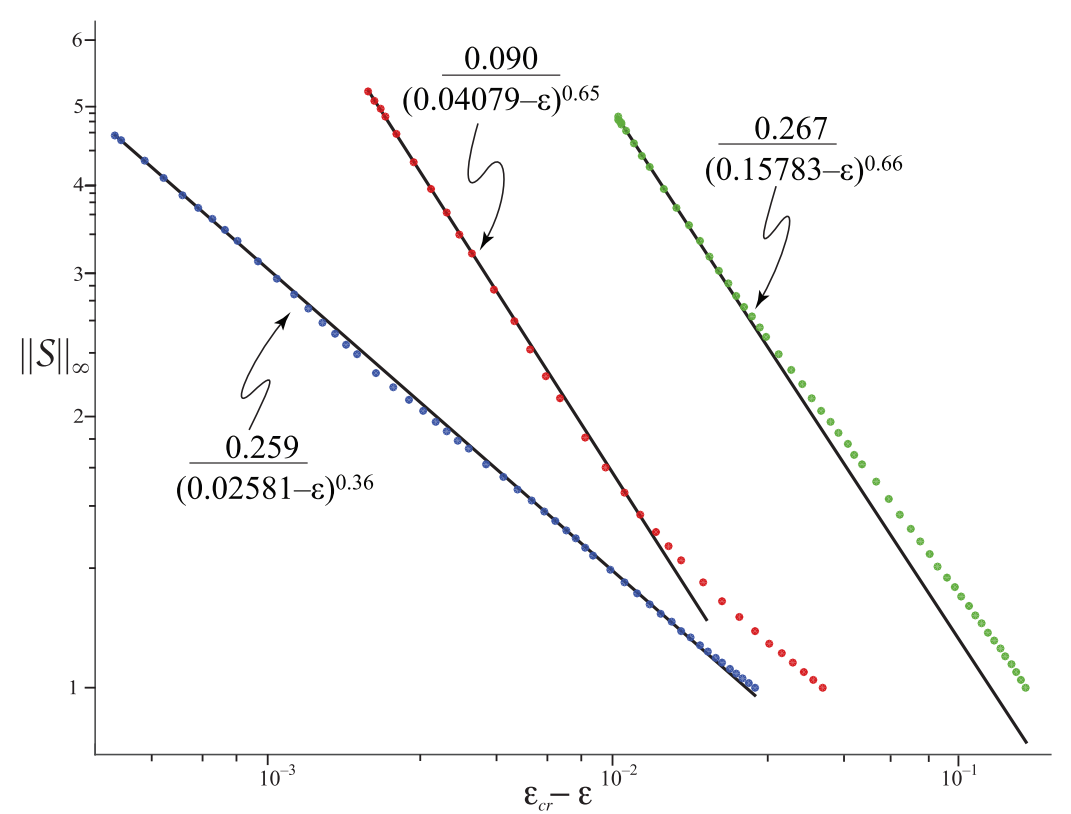

Figure 11. Blowup of $\mathcal{S}$ for the $\omega=\left(\sigma-1, \sigma^{2}-1\right)$ invariant torus for three volume-preserving maps. The bottom (blue) curve corresponds to the standard volume-preserving map. The upper and middle curves are for the $A B C$ map (32). The middle (red) curve is for $B=2 \varepsilon$ and the top (green) is for $B=\varepsilon^{2}$. The horizontal scale is based on the best estimate of $\varepsilon_{\text {cr }}$ from (36). 
Table 2

Estimates of $\varepsilon_{c r}$ for tori of (26) with 16 different rotation vectors in $\mathbb{Q}(\sigma)$ determined by the Farey paths in column two. The third and forth columns give $\varepsilon_{c r}(\omega)$ computed by the residue method (using orbits up to period 100,000) and the Fourier method from the singular value fit (36), respectively. The numbers in parentheses indicate the estimated error in the least significant digit of $\varepsilon_{c r}$. The last column is the fit to $\beta$ from (36).

\begin{tabular}{|c|c|c|c|c|}
\hline$\omega$ & Farey Path & $\varepsilon_{c r}^{R}(\omega)$ & $\varepsilon_{c r}^{s}(\omega)$ & $\bar{\beta}$ \\
\hline$(0.3247,0.7549)$ & $l l \bar{r}$ & $0.0258(1)$ & $0.02581(2)$ & 0.36 \\
\hline$(0.5278,0.8286)$ & llrrrl $\bar{r}$ & $0.03226(8)$ & $0.0321(1)$ & 0.40 \\
\hline$(0.2068,0.8439)$ & $l l r r l \bar{r}$ & $0.01741(2)$ & $0.01728(2)$ & 0.38 \\
\hline$(0.1054,0.6753)$ & llrrll $\bar{r}$ & $0.01255(3)$ & $0.012324(5)$ & 0.54 \\
\hline$(0.1850,0.4302)$ & $l l r l \bar{r}$ & $0.01242(1)$ & $0.01195164(3)$ & 0.49 \\
\hline$(0.1294,0.3008)$ & llrlrl $\bar{r}$ & $0.00671(4)$ & $0.0067(4)$ & 1.06 \\
\hline$(0.4809,0.6370)$ & $l l r l l \bar{r}$ & $0.02141(4)$ & $0.0220(1)$ & 0.61 \\
\hline$(0.2451,0.5698)$ & llrllle $\bar{r}$ & $0.01754(2)$ & $0.01750(3)$ & 0.46 \\
\hline$(0.5698,0.3247)$ & $r r l \bar{r}$ & $0.03642(5)$ & $0.03638(3)$ & 0.43 \\
\hline$(0.6992,0.5278)$ & rrlrrlō & $0.03404(6)$ & $0.03367(2)$ & 0.42 \\
\hline$(0.3630,0.2068)$ & $r r l r l \bar{r}$ & $0.01051(3)$ & $0.01017(3)$ & 0.54 \\
\hline$(0.4302,0.1054)$ & rrlrlli $\bar{r}$ & $0.013420(6)$ & $0.01275(8)$ & 0.57 \\
\hline$(0.7549,0.1850)$ & rrllo & $0.027469(6)$ & $0.02742(6)$ & 0.85 \\
\hline$(0.8286,0.1294)$ & $\operatorname{rrllrl} \bar{r}$ & $0.01323(2)$ & $0.0128(3)$ & 0.77 \\
\hline$(0.8439,0.4809)$ & rrllli & $0.02682(9)$ & $0.02630(6)$ & 0.66 \\
\hline$(0.6753,0.2451)$ & rrlllli $\bar{r}$ & $0.03527(2)$ & $0.03503(6)$ & 0.69 \\
\hline
\end{tabular}

no significant change in the estimate between $N_{\max }=2^{11}$ and $N_{\max }=2^{12}$. We also note that the error estimate for $N_{\max }=2^{9}$ is certainly too small.

The results from the singular value method can be compared to those we obtained using Greene's residue criterion for (26) from [FM13]. To do this, we generated sixteen rotation vectors in $\mathbb{Q}(\sigma)$ by adding an infinite tail of $r$ symbols to the binary sequences of rational vectors from level 6 of the generalized Farey tree, restricted to the unit square; recall section 3. The critical $\varepsilon$ values for the corresponding tori were estimated using both the singular value method and Greene's criterion for orbits up to period 100,000 with a threshold residue $R_{t h}=$ 5.0. The reasonable agreement between these results, shown in Table 2, provides numerical evidence for the validity of both methods, although the error estimates generally appear too small in both cases.

Similar results for the tori with the same sixteen rotation vectors for the ABC map (32) are summarized in Table 3. Since this map is not reversible, we cannot compare these results with Greene's residue criterion. The estimated errors in the fits to (36) are of similar orders of magnitude as that found in Table 2, though as before, this is likely an underestimate of the true error. We also found that the critical parameter for the $l l \bar{r}$ torus of the ABC map varies smoothly with the parameter $B$. For example, Figure 12 shows $\varepsilon_{c r}$ as a function of $b$, where $B=b \varepsilon$. As $b$ grows the perturbation to the system increases, and, as expected, the torus becomes increasingly fragile.

6. Conclusions. In this paper we applied a Fourier-based, quasi-Newton algorithm to efficiently and accurately compute the conjugacy for rotational tori in volume-preserving maps with two angles and one action. When the map is reversible, the tori we found satisfy the 
Table 3

Estimates of $\varepsilon_{c r}$ for tori of (32) with 16 different rotation vectors in $\mathbb{Q}(\sigma)$. The third and fifth columns give $\varepsilon_{c r}(\omega)$ computed by the singular value method for $B=2 \varepsilon$ and $B=\varepsilon^{2}$, respectively. The numbers in parentheses indicate the estimated error in the least significant digit of $\varepsilon_{c r}$. The fourth and sixth columns give the fit to $\beta$ from (36).

\begin{tabular}{|c|c|c|c|c|c|}
\hline \multirow[b]{2}{*}{$\omega$} & \multirow[b]{2}{*}{ Farey Path } & \multicolumn{2}{|c|}{$B=2 \varepsilon$} & \multicolumn{2}{|c|}{$B=\varepsilon^{2}$} \\
\hline & & $\varepsilon_{c r}(\omega)$ & $\beta$ & $\varepsilon_{c r}(\omega)$ & $\beta$ \\
\hline$(0.3247,0.7549)$ & $l l \bar{r}$ & $0.04079(7)$ & 0.65 & $0.15783(9)$ & 0.63 \\
\hline$(0.5278,0.8286)$ & llrrrl $\bar{r}$ & $0.0332(1)$ & 0.40 & $0.1146(4)$ & 0.47 \\
\hline$(0.2068,0.8439)$ & llrrl $\bar{r}$ & $0.0380(3)$ & 0.86 & $0.142(3)$ & 0.86 \\
\hline$(0.1054,0.6753)$ & $\operatorname{llr} r l l \bar{r}$ & $0.0239(1)$ & 0.52 & $0.153(1)$ & 0.56 \\
\hline$(0.1850,0.4302)$ & $l l r l \bar{r}$ & $0.0693(1)$ & 0.36 & $0.2876(2)$ & 0.30 \\
\hline$(0.1294,0.3008)$ & llrlrl $\bar{r}$ & $0.0655(2)$ & 0.45 & $0.2701(6)$ & 0.59 \\
\hline$(0.4809,0.6370)$ & $l l r l l \bar{r}$ & $0.0396(7)$ & 0.69 & $0.1815(2)$ & 0.60 \\
\hline$(0.2451,0.5698)$ & llrllli $\bar{r}$ & $0.06237(5)$ & 0.49 & $0.23586(1)$ & 0.61 \\
\hline$(0.5698,0.3247)$ & $r r l \bar{r}$ & $0.08315(5)$ & 0.47 & $0.1254(5)$ & 0.99 \\
\hline$(0.6992,0.5278)$ & rrlrrl $\bar{r}$ & $0.049562(4)$ & 0.39 & $0.09613(9)$ & 0.68 \\
\hline$(0.3630,0.2068)$ & $r r l r l \bar{r}$ & $0.10383(4)$ & 0.43 & $0.1863(8)$ & 0.52 \\
\hline$(0.4302,0.1054)$ & rrlrlle & $0.0654(5)$ & 0.46 & $0.1248(3)$ & 0.70 \\
\hline$(0.7549,0.1850)$ & rrller & $0.0389(2)$ & 0.54 & $0.1000(1)$ & 0.25 \\
\hline$(0.8286,0.1294)$ & rrllrl $\bar{r}$ & $0.0190(6)$ & 0.57 & $0.0582(6)$ & 0.29 \\
\hline$(0.8439,0.4809)$ & $\operatorname{rrlll} \bar{r}$ & $0.0382(1)$ & 0.43 & $0.0618(7)$ & 0.65 \\
\hline$(0.6753,0.2451)$ & rrlllli $\bar{r}$ & $0.0659(1)$ & 0.33 & $0.1134(4)$ & 0.57 \\
\hline
\end{tabular}

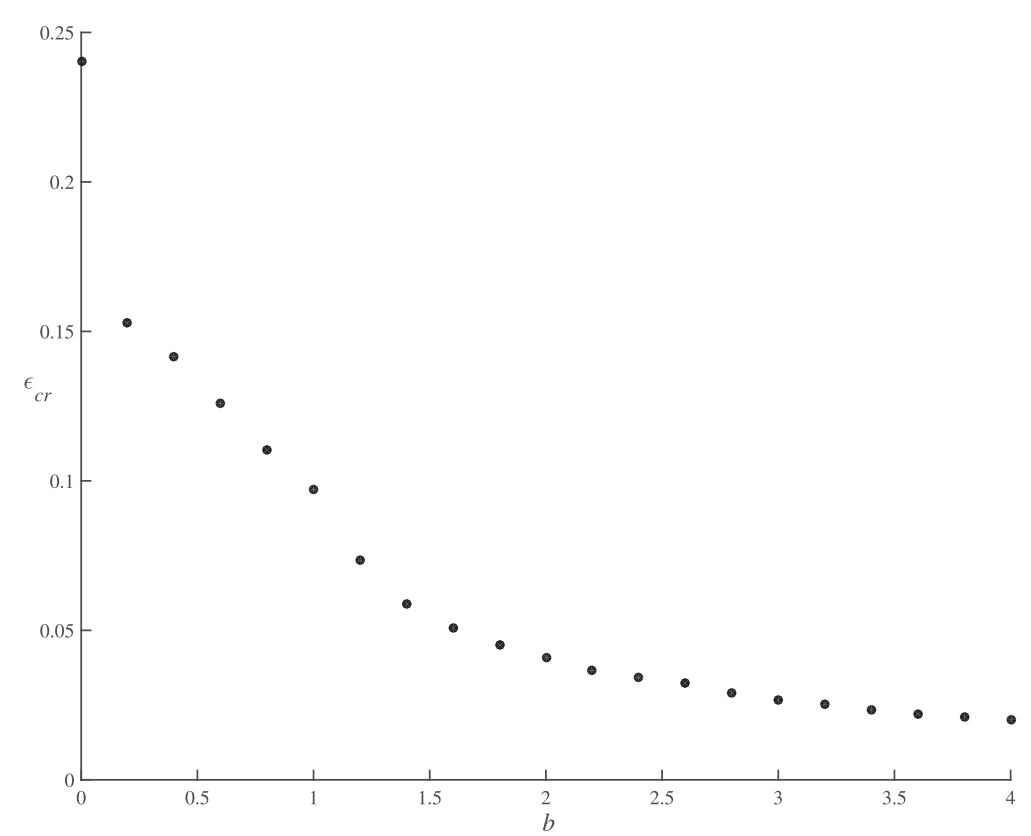

Figure 12. Estimate of $\varepsilon_{c r}$ as a function of $b$ for the $l l \bar{r}$ torus in the $A B C$ map (32) with $B=b \varepsilon$, computed by the singular value method.

expected symmetry properties and are the limits of sequences of symmetric periodic orbits like those we found in [FM13]. The Fourier method, however, also applies to nonreversible maps and, as an example, we showed that it can be used to compute tori for the ABC map. 
Reasonably accurate estimates (4-5 significant figures) for the parameters at which a torus is critical were obtained under the hypothesis that the local singular values of the derivative of the conjugacy blow up as a power-law at criticality. The computations of $\varepsilon_{c r}$, however, are unfortunately not as accurate as those obtained using Greene's residue method for areapreserving maps. Part of the reason for this is probably intrinsic to the number theory of the spiral mean and of Diophantine vectors more generally, which are not as regular as the noble numbers that give the most robust invariant circles in the area-preserving case. It is not clear to us how to make significant progress on this problem.

The patterns of peaks of the local singular values of the conjugacy show local distortions in the torus that eventually should be responsible for tearing it apart. Examination of these near-critical conjugacies may give further insight into the destruction of tori, and may provide insight into the topological structure of invariant sets that replace the tori when $\varepsilon>\varepsilon_{c r}$, if any such sets exist. Barring a generalization of Aubry-Mather theory to the volume-preserving case - which seems difficult since we know of no variational formulation for these maps - it would be nice to develop an anti-integrable theory for maps on $\mathbb{T}^{2} \times \mathbb{R}$ that could show the existence of invariant sets analogous to cantori.

Finally, it would be interesting to extend this method to compute nonrotational tori, as well as tori for incompressible flows and higher-dimensional, volume-preserving maps.

Acknowledgments. Useful conversations with Holger Dullin, Robert Easton, and Keith Julien are gratefully acknowledged. We would also like to thank Timothy Blass and Rafael de la Llave for discussing their results in advance of publication. They are independently developing, and obtaining a rigorous justification for, a similar method [BdlL15].

\section{REFERENCES}

[Aub95] S. J. AubRY, Anti-integrability in dynamical and variational problems, Phys. D, 86 (1995), pp. 284296, http://dx.doi.org/10.1016/0167-2789(95)00109-H.

[BdlL15] T. Blass AND R. DE la Llave, KAM Theory for Volume-Preserving Maps, manuscript.

[Cas57] J. W. S. CAssels, An Introduction to Diophantine Approximation, Cambridge University Press, Cambridge, 1957.

[CdlL10a] R. Calleja and R. De la Llave, Computation of the breakdown of analyticity in statistical mechanics models: Numerical results and a renormalization group explanation, J. Stat. Phys., 141 (2010), pp. 940-951, http://dx.doi.org/10.1007/s10955-010-0085-7.

[CdlL10b] R. Calleja AND R. DE la Llave, A numerically accessible criterion for the breakdown of quasi-periodic solutions and its rigorous justificaition, Nonlinearity, 23 (2010), pp. 2029-2058, http://dx.doi.org/10.1088/0951-7715/23/9/001.

[CFP96] J. H. E. Cartwright, M. Feingold, And O. Piro, Chaotic advection in three dimensional unsteady incompressible laminar flow, J. Fluid Mech., 316 (1996), pp. 259-284, http://dx.doi.org/10.1017/ S0022112096000535.

[CS90] C.-Q. Cheng AND Y.-S. Sun, Existence of invariant tori in three-dimensional measurepreserving mappings, Celestial Mech. Dynam. Astronom., 47 (1990), pp. 275-292, http://dx.doi.org/10.1007/BF00053456.

$\left[\mathrm{DFG}^{+} 86\right]$ T. Dombre, U. Frisch, J. M. Greene, M. Hénon, A. Mehr, And A. M. Soward, Chaotic streamlines in the ABC flows, J. Fluid Mech., 167 (1986), pp. 353-391, http://dx.doi.org/ 10.1017/S0022112086002859.

[DM12] H. R. Dullin AND J. D. Meiss, Resonances and twist in volume-preserving mappings, SIAM J. Appl. Dyn. Syst., 11 (2012), pp. 319-349, http://dx.doi.org/10.1137/110846865. 
[FdllS09] E. Fontich, R. De la Llave, And Y. Sire, Construction of invariant whiskered tori by a parameterization method. Part I: Maps and flows in finite dimensions, J. Differential Equations, 268 (2009), pp. 3136-3213, http://dx.doi.org/10.1016/j.jde.2009.01.037

[FM13] A. M. Fox AND J. D. MEISs, Greene's residue criterion for the breakup of invariant tori of volume-preserving maps, Phys. D, 243 (2013), pp. 45-63, http://www.sciencedirect.com/science/ article/pii/S016727891200245X.

[FM14] A. M. Fox AND J. D. MeIss, Critical invariant circles in asymmetric and multiharmonic generalized standard maps, Commun. Nonlinear Sci. Numer. Simul., 19 (2014), pp. 1004-1026, http://dx.doi.org/10.1016/j.cnsns.2013.07.028.

[FO88a] J. M. Finn And E. OTt, Chaotic flows and fast magnetic dynamos, Phys. Fluids, 31 (1988), pp. 2992-3011, http://dx.doi.org/10.1063/1.866956.

[FO88b] J. M. Finn AND E. OTt, Chaotic flows and magnetic dynamos, Phys. Rev. Lett., 60 (1988), pp. 760-763, http://link.aps.org/doi/10.1103/PhysRevLett.60.760.

[FP88] L. P. Feingold, M. Kadanoff And O. Piro, Passive scalars, three-dimensional volumepreserving maps, and chaos, J. Stat. Phys., 50 (1988), pp. 529-565, http://dx.doi.org/ 10.1007/BF01026490.

[Gre79] J. M. GreEne, A method for determining a stochastic transition, J. Math. Phys, 20 (1979), pp. 1183-1201, http://dx.doi.org/10.1063/1.524170.

[HdlL07] A. HARO AND R. DE LA Llave, A parameterization method for the computation of invariant tori and their whiskers in quasi-periodic maps: Explorations and mechanisms for the breakdown of hyperbolicity, SIAM J. Appl. Dyn. Syst., 6 (2007), pp. 142-207, http://dx.doi.org/doi:10.1137/050637.

[Hdils12] G. Huguet, R. De la Llave, and Y. Sire, Computation of whiskered invariant tori and their associated manifolds: New fast algorithms, Discrete Contin. Dyn. Syst. Ser. A, 32 (2012), pp. 1309-1353, http://dx.doi.org/10.3934/dcds.2012.32.1309.

[JLM08] J. JuAng, M.-C. LI, AND M. MALKIn, Chaotic difference equations in two variables and their multidimensional perturbations, Nonlinearity, 21 (2008), pp. 1019-1040, http://dx.doi.org/10.1088/0951-7715/21/5/007.

[KO86] S.-H. KIM AND S. OstLund, Simultaneous rational approximations in the study of dynamical systems, Phys. Rev. A (3), 34 (1986), pp. 3426-3434, http://dx.doi.org/10.1103/PhysRevA. 34.3426 .

[LS94] J. LIU AND Y.-S. Sun, Chaotic motion of comets in near-parabolic orbit: Mapping approaches, Celestial Mech. Dynam. Astronom., 60 (1994), pp. 3-28, http://dx.doi.org/10.1007/BF00693090.

[Mei12] J. MeIss, The destruction of tori in volume-preserving maps, Commun. Nonlinear Sci. Numer. Simul., 17 (2012), pp. 2108-2121, http://dx.doi.org/10.1016/j.cnsns.2011.04.014.

[Mei15] J. D. Meiss, Thirty years of turnstiles and transport, Chaos, 25 (2015), 097602, http://dx.doi.org/ 10.1063/1.4915831.

[MLO07] S. W. Meier, R. M. Lueptow, And J. M. Ottino, A dynamical systems approach to mixing and segregation of granular materials in tumblers, Adv. Phys., 56 (2007), pp. 757-827, http://dx.doi.org/10.1080/00018730701611677.

[Mos66] J. Moser, On the theory of quasiperiodic motions, SIAM Rev., 8 (1966), pp. 145-172, http://epubs.siam.org/sirev/resource/1/siread/v8/i2/p145_s1.

[MW99] I. MEzIĆ And S. Wiggins, A method for visualization of invariant sets of dynamical systems based on the ergodic partition, Chaos, 9 (1999), pp. 213-218, http://authors.library.caltech. edu/1939/1/MEZchaos99a.pdf.

[PF88] O. Piro And M. Feingold, Diffusion in three-dimensional Liouvillian maps, Phys. Rev. Lett., 61 (1988), pp. 1799-1802, http://dx.doi.org/10.1103/PhysRevLett.61.1799.

[Ste96] I. StewART, Tales of a neglected number, Sci. Am., 274 (1996), pp. 102-103, http://dx.doi. org/10.1038/scientificamerican0696-102.

[TH85] A. Thyagaraja AND F. HAAs, Representation of volume-preserving maps induced by solenoidal vector fields, Phys. Fluids, 28 (1985), pp. 1005-1007, http://dx.doi.org/10.1063/1.865093.

[Tre00] L. N. Trefethen, Spectral Methods in MATLAB, Software, Environ., Tools, SIAM, Philadelphia, 2000.

[Xia92] Z. XIA, Existence of invariant tori in volume-preserving diffeomorphisms, Ergodic Theory Dynam. Systems, 12 (1992), pp. 621-631, http://dx.doi.org/10.1017/S0143385700006969. 